\title{
The final core collapse of pulsational pair instability supernovae
}

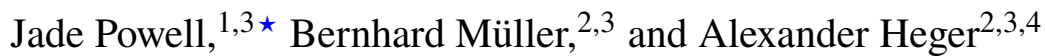 \\ ${ }^{1}$ Centre for Astrophysics and Supercomputing, Swinburne University of Technology, Hawthorn, VIC 3122, Australia. \\ ${ }^{2}$ Monash Centre for Astrophysics, School of Physics and Astronomy, Monash University, VIC 3800, Australia. \\ ${ }^{3}$ ARC Centre of Excellence for Gravitational Wave Discovery (OzGrav), Melbourne, Australia. \\ ${ }^{4}$ ARC Centre of Excellence for Astrophysics in Three Dimensions (ASTRO-3D), Australia.
}

1 March 2021

\begin{abstract}
We present 3D core-collapse supernova simulations of massive Pop-III progenitor stars at the transition to the pulsational pair instability regime. We simulate two progenitor models with initial masses of $85 \mathrm{M}_{\odot}$ and $100 \mathrm{M}_{\odot}$ with the LS220, SFHo, and SFHx equations of state. The $85 \mathrm{M}_{\odot}$ progenitor experiences a pair instability pulse coincident with core collapse, whereas the $100 \mathrm{M}_{\odot}$ progenitor has already gone through a sequence of four pulses 1,500 years before collapse in which it ejected its $\mathrm{H}$ and $\mathrm{He}$ envelope. The $85 \mathrm{M}_{\odot}$ models experience shock revival and then delayed collapse to a black hole (BH) due to ongoing accretion within hundreds of milliseconds. The diagnostic energy of the incipient explosion reaches up to $2.7 \times 10^{51} \mathrm{erg}$ in the SFHx model. Due to the high binding energy of the metal core, BH collapse by fallback is eventually unavoidable, but partial mass ejection may be possible. The $100 \mathrm{M}_{\odot}$ models have not achieved shock revival or undergone $\mathrm{BH}$ collapse by the end of the simulation. All models exhibit relatively strong gravitational-wave emission both in the high-frequency g-mode emission band and at low frequencies. The SFHx and SFHo models show clear emission from the standing accretion shock instability. For our models, we estimate maximum detection distances of up to $\sim 46 \mathrm{kpc}$ with LIGO and $\sim 850 \mathrm{kpc}$ with Cosmic Explorer.
\end{abstract}

Key words: transients: supernovae - gravitational waves

\section{INTRODUCTION}

Core-collapse supernovae (CCSNe) occur when the iron cores of stars above $\sim 8 \mathrm{M}_{\odot}$ reach their effective Chandrasekhar mass and collapse until they reach nuclear density. As the core rebounds elastically, a shock wave is launched outwards which quickly loses energy and stalls. For a successful explosion, the shock must be revived. According to the current paradigm, shock revival is achieved by neutrino heating in most CCSNe, but in rare cases of unusually energetic "hypernovae" some form of magnetohydrodynamic mechanism may play a key role as well (for a review, see Janka 2012).

Due to the complicated nature of CCSNe, simulations are essential for understanding their explosion dynamics, observable multimessenger emission, and remnant properties. Multi-dimensional simulations of neutrino-driven CCSNe have advanced rapidly in recent years and are starting to reveal the systematics of explosion and remnant properties, however, the full parameter space of selfconsistent 3D explosions has not yet been fully explored (see Müller 2020; Burrows \& Vartanyan 2020 for recent reviews).

CCSNe are the birth places of neutron stars and stellar mass black holes (BHs), which are the primary sources for gravitational-wave (GW) detectors such as Advanced LIGO (The LIGO Scientific Collaboration et al. 2015), Advanced Virgo (Acernese \& et al. 2015) and KAGRA (Somiya 2012). The birth masses, spins and kicks of these compact objects cannot be understood without CCSN simulations.

Recent GW detections of high-mass BHs have drawn particular

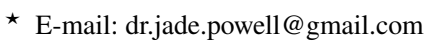

attention to the upper end of the supernova progenitor-mass distribution and the transition to different explosion regimes. Instead of proceeding with advanced nuclear burning stages up to the formation of an iron core, stars with helium cores in the range $\sim 50-150 \mathrm{M}_{\odot}$ are believed to become unstable to electron-positron pair production (Fowler \& Hoyle 1964; Barkat et al. 1967), which may completely unbind the star resulting in a pair-instability supernova explosion, leaving no remnant behind (Heger \& Woosley 2002; Heger et al. 2003; Chen et al. 2014; Kozyreva et al. 2017). Pulsational pairinstability supernovae are stars with helium core masses in the range 230-50 M $\odot$ (Heger \& Woosley 2002; Heger et al. 2003). They also experience pair instability that results in pulsations that eject material, but as the energy of the pulsations is lower, the star is not completely disrupted (Heger et al. 2003; Woosley 2017). These stars are then expected to undergo a regular core collapse that may result in a supernova or a gamma-ray burst (GRB) (Woosley et al. 2007). As a result, stars in this mass range should form BHs with masses in the range of $30-45 \mathrm{M}_{\odot}$; above that one expects a "mass gap" from the complete disruption of the star by a successful pair instability supernova (Belczynski et al. 2016; Stevenson et al. 2019). However, optical observations of binary systems (Liu et al. 2019) as well as GW signals from binary BH mergers by LIGO and Virgo (The LIGO Scientific Collaboration et al. 2020) have recently discovered BHs with suggested or most likely masses in this pair-instability mass gap. Simulations of the explosions of very massive BH-forming models are important to provide further insights into the unexpected masses found by GW observations.

CCSNe are also of interest for GW astronomy as targets in their own right. As the sensitivity of GW detectors increases, they will be- 
gin to detect not only binary mergers but also other lower-amplitude sources of GWs such as CCSNe. Accurate knowledge of the GW emission from CCSNe will be essential for detection and parameter estimation. The GW signal from rotational core bounce has already been well covered in the literature (e.g., Dimmelmeier et al. 2008; Abdikamalov et al. 2014; Fuller et al. 2015; Richers et al. 2017). In the non-rotating case, the GW emission from the post-bounce phase has been studied using self-consistent 3D simulations by many groups (Andresen et al. 2017, 2019, 2020; Kuroda et al. 2016, 2017; Kuroda et al. 2018; Radice et al. 2019; Powell \& Müller 2019, 2020; Mezzacappa et al. 2020; Pan et al. 2020). The structure of the GW emission has shown common features in different simulations from recent years. The dominant emission feature in the GW emission is due to the quadrupolar surface $\mathrm{f} / \mathrm{g}$-mode ${ }^{1}$ of the proto-neutron star (PNS), which produces GW frequencies rising in time from a few hundred $\mathrm{Hz}$ up to a few $\mathrm{kHz}$ (Müller et al. 2012; Sotani et al. 2017; Morozova et al. 2018; Torres-Forné et al. 2018; Kuroda et al. 2018; Torres-Forné et al. 2019). In addition, some models (Kuroda et al. 2016, 2017; Andresen et al. 2017; Powell \& Müller 2020; Mezzacappa et al. 2020) exhibit low-frequency GW emission due to the standing accretion shock instability (SASI; Blondin et al. 2003; Blondin \& Mezzacappa 2006; Foglizzo et al. 2007). In rapidly rotating models, very strong GW emission can also occur during the post-bounce phase due to a corotation instability (Takiwaki \& Kotake 2018). The emerging understanding of the GW emission features has led to the formulation of universal relations for the GW emission (Torres-Forné et al. 2019) and paved the way for phenomenological modelling for CCSN signals (Astone et al. 2018). Further work is still needed, however, to extend these models to fully explore CCSN GW signals from across the progenitor parameter space. The majority of 3D simulations that include GW emission are for progenitor stars below $30 \mathrm{M}_{\odot}$. In this paper, we perform simulations of highmass Population III (Pop-III) stars in the pulsational pair instability regime to expand the parameter space coverage of $3 \mathrm{D}$ simulations and to provide further insights into the massive and very massive star remnant $\mathrm{BH}$ population.

A small number of studies have already focused on failed or partially successful CCSNe with $\mathrm{BH}$ formation in the regime of highmass progenitors, but they have not extensively investigated their GW emission. Kuroda et al. (2018) simulated the collapse to a BH of a $70 \mathrm{M}_{\odot}$ progenitor, and found very large GW amplitudes as convection dominated over SASI in their model. Strong SASI was also found in the simulations of a $70 \mathrm{M}_{\odot}$ progenitor by Shibagaki et al. (2020, 2021). No such phenomenon was reported in the $3 \mathrm{D}$ simulations of $\mathrm{BH}$ formation in a $40 \mathrm{M}_{\odot}$ progenitor by Chan et al. $(2018,2020)$ despite powerful SASI activity with a clear imprint on the neutrino signal (Müller 2019), but no further analysis of the GW emission has been carried out for these models. The GW emission prior to $\mathrm{BH}$ collapse also remains of modest amplitude in the recent 3D models of $\mathrm{BH}$ collapse for a $40 \mathrm{M}_{\odot}$ progenitor by Pan et al. (2020), though an earlier 2D study (Pan et al. 2018) did show enhanced GW emission shortly before collapse in some cases. The $40 \mathrm{M}_{\odot}$ models of Pan et al. (2020) with different rotation rates were, however, noteworthy for predicting very high $\mathrm{GW}$ frequencies of up to $\sim 3,000 \mathrm{~Hz}$ before $\mathrm{BH}$ formation. It is important to determine if this is a robust prediction because of the strongly frequency-dependent sensitivity of GW

\footnotetext{
1 The mode that sets the dominant emission frequency can change character from a g-mode to an f-mode (Morozova et al. 2018; Sotani \& Takiwaki 2020). For the sake of simplicity, we often refer to its frequency simply as the g-mode frequency even though its precise character at a given time is not known.
}

detectors. The recent work on $\mathrm{BH}$ formation in $40 \mathrm{M}_{\odot}$ and $75 \mathrm{M}_{\odot}$ progenitors by Walk et al. (2020) did not discuss GW emission, but pointed out an interesting feature in $\mathrm{BH}$-forming models that could lead to very strong GW emission. Due to the extreme recession of the shock, the $l=2$ quadrupole mode of the SASI becomes unstable and dominates the $l=1$ mode during some phases of the evolution. A strong $l=1$ mode already gives a relatively strong GW signal because of a finite admixture of $l=2$ density perturbations that are seen in GWs. If the dominant SASI mode has $l=2$ to begin with, the signal could be much stronger.

Beyond the high-mass end of the "mass gap" Fryer et al. (2001) simulated the collapse of a rapidly-rotating $300 \mathrm{M}_{\odot}$ Pop-III model. In that simulation a $50 \mathrm{M}_{\odot}$ rapidly-rotating core formed during collapse that was held up by trapped neutrinos and susceptible to secular triaxial instabilities that could grow on a time-scale shorter than the collapse time, potentially also being a powerful GW source with $h_{+} \sim 10^{-21}$ at $1 \mathrm{Gpc}$.

If there is strongly enhanced GW emission prior to the final collapse, BH-forming massive stars may be observable in GWs at larger distances than normal CCSNe. Such a strong GW signal could provide clues about the neutron star mass and radius before the final collapse, e.g., through the maximum g-mode frequency. A GW detection from such an event would provide valuable complementary information about $\mathrm{BH}$ formation to optical surveys for disappearing massive stars (Smartt 2015; Gerke et al. 2015; Adams et al. 2017a,b). In this context, it is intriguing that recent simulations (Chan et al. 2018; Kuroda et al. 2018; Ott et al. 2018; Pan et al. 2020) suggested that shock expansion could occur in massive stars just before $\mathrm{BH}$ formation; and this "hiccup" may even give rise to an observable transient (Moriya et al. 2019). Further simulations of BH forming models are needed to determine when to expect stars to collapse quietly and when there may be an early shock revival before collapse followed by extensive fallback.

In this paper, we aim to further clarify the fate and GW signatures of the most massive CCSN progenitors. We perform 3D simulations of BH forming stellar collapse with three different equations of state (EoS) that result in different maximum neutron star masses and $\mathrm{BH}$ formation times. In addition to the LS220 EoS (Lattimer \& Swesty 1991) used in our previous simulations (Powell \& Müller 2019, 2020), we will use two EoS (SFHx and SFHo, Steiner et al. 2013) with a higher maximum neutron star mass and smaller neutron star radii. The progenitor models are $85 \mathrm{M}_{\odot}$ and $100 \mathrm{M}_{\odot}$ Pop-III stars. These masses are higher than any of the other recent self-consistent 3D simulations of $\mathrm{BH}$ forming models and probe the lower end of the pulsational pair instability regime.

Using these models, we investigate the possibility of shock expansion before $\mathrm{BH}$ formation for a wider range of progenitors and EoS and, where applicable, examine the effects of the different EoS on the explosion dynamics. We then analyse the detectability of the GW signals. We determine the maximum detection distances for our models using simulated design sensitivity Gaussian noise for the LIGO, Einstein Telescope (Punturo et al. 2010), and Cosmic Explorer (Abbott et al. 2017) detectors, and we also discuss the detectability of features in the time-frequency structure of the signal in noisy spectrograms.

The outline of our paper is as follows: In Section 2, we present the two progenitor models. In Section 3, we provide details on the numerical methods and the setup of our simulations. In Section 4 , we analyse the dynamics of our models. We describe the features of the GW emission in Section 5, and present a discussion and conclusions in Section 6. 


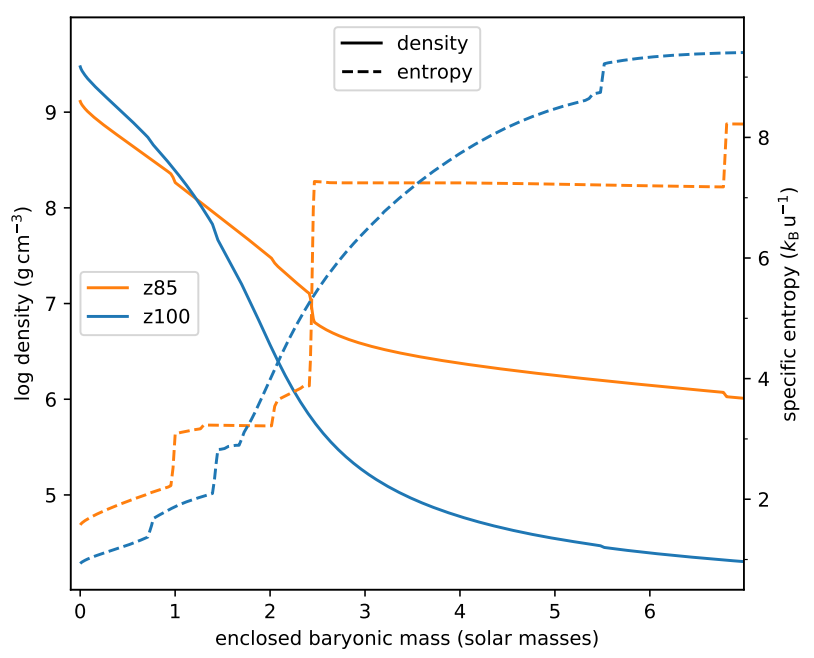

Figure 1. Profiles of the density (solid lines) and specific entropy (dashed lines) as a function of mass coordinate, $m$, for the progenitor models $z 85$ (orange) and $z 100$ (blue) at the onset of core collapse, defined as the first model in which the infall velocity exceeds $900 \mathrm{~km} \mathrm{~s}^{-1}$. Model $z 85$ has a large jump in entropy and density between the silicon core and the convective $\mathrm{O}$ shell at a mass coordinate of $m=2.4 \mathrm{M}_{\odot}$. In model $\mathrm{z} 100$, traces of remaining oxygen burn between $m=1.9 \mathrm{M}_{\odot}$ and $m=5.5 \mathrm{M}_{\odot}$ leading to a smooth shallow entropy and density gradient instead. Unlike model z85, the flat profile above $m=5.5 \mathrm{M}_{\odot}$ is not convective and not powered by nuclear burning. The unusual structure is a result of the final pair-instability pulse (Figures 5 and 6).

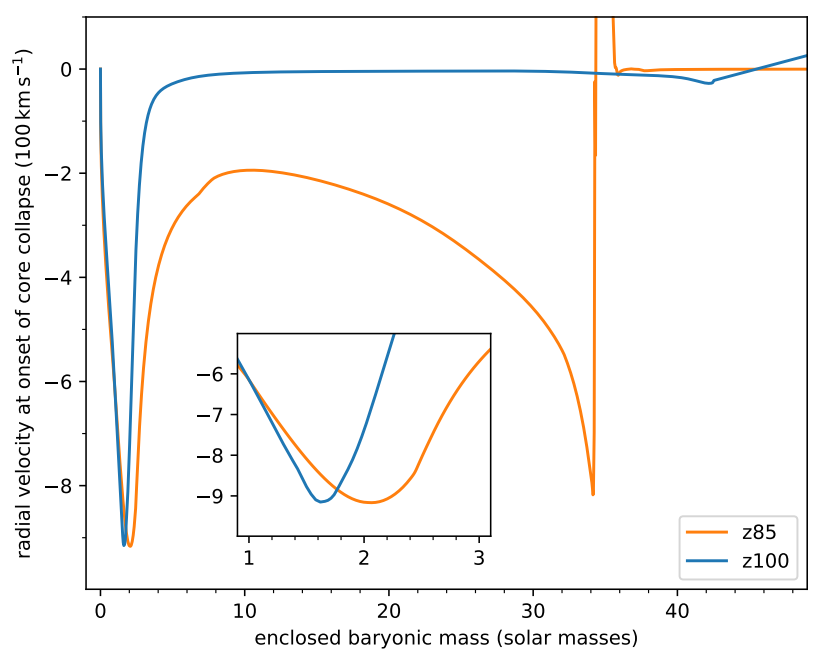

Figure 2. Radial velocity profile of models z85 (orange) and z100 (blue) as a function of mass coordinate $m$ at onset of core collapse, defined as the first model in which the infall velocity exceeds $900 \mathrm{~km} \mathrm{~s}^{-1}$. The insert magnifies the locations of the peak infall velocities, which are at mass coordinates of $2.1 \mathrm{M}_{\odot}$ and $1.6 \mathrm{M}_{\odot}$, respectively. In model z85, the entire $\mathrm{CO}$ core of $\sim 34 \mathrm{M}_{\odot}$ is collapsing due to pair instability occurring at iron core collapse, whereas in model z100 a much slower contracting core is seen out to $m \sim 42.5 \mathrm{M}_{\odot}$. In model $z 100$, the entropy in the core is much larger in the aftermath of the preceding pair-instability pulses and a much smaller $\left(1.6 \mathrm{M}_{\odot}\right)$ homologouslycollapsing iron core results than in model z85 $\left(2.1 \mathrm{M}_{\odot}\right)$.

\section{PROGENITOR MODELS}

We simulate the collapse of two zero-metallicity (Pop-III) progenitor models, z85 and z100 (Heger \& Woosley 2010), with zero-age main sequence masses of $85 \mathrm{M}_{\odot}$ and $100 \mathrm{M}_{\odot}$, respectively. Both models are located close to the lower boundary of the pulsationalpair instability regime. The progenitor models have been evolved up to collapse using the stellar evolution code KePLER (Weaver et al. 1978; Rauscher et al. 2002). Core density and entropy profiles (Figure 1 ) and radial velocity profiles (Figure 2 ) of the two progenitor models reveal substantial structural differences. In particular, model z85 largely follows a typical massive star evolution path (Figure 3; Woosley et al. 2002) but encounters oscillatory-unstable oxygen shell burning (Figure 4 ) and eventually pair instability during iron core collapse (Figure 2), whereas the final structure of model z100 is heavily affected by pair-instability pulses long before the final collapse (Figures 5 and 6 ).

\subsection{Progenitor Model z85}

At first glance, the $85 \mathrm{M}_{\odot}$ model exhibits a classical structure typical of most CCSN progenitors, just with a very massive core. The lowentropy core inside the first convective shell has as mass of $2.43 \mathrm{M}_{\odot}$, and both the jumps in specific entropy and density between the core and the surrounding shell are extremely well pronounced, with a huge shell specific entropy of $7.3 k_{\mathrm{B}} \mathrm{u}^{-1}$, where $k_{\mathrm{B}}$ is the Boltzmann constant and $\mathrm{u}$ is the atomic mass unit. Both the Ertl criterion (Ertl et al. 2016) and the compactness criterion (O'Connor \& Ott 2011) firmly predict $\mathrm{BH}$ formation for this model due to the rather extreme values of the structural parameters $M_{4}=2.43$ and $\mu_{4}=2.90$ and a very high compactness of $\xi_{2.5}=0.86$.

A closer examination of the evolution of the model and its structure and composition at collapse reveal noticeable differences from normal CCSN progenitors. Silicon core burning proceeds while oxygen burning above continues almost unaltered due to the large $\sim 2.5 \mathrm{M}_{\odot}$ silicon core. At onset of core collapse, defined as the first model in which the infall velocity exceeds $900 \mathrm{~km} \mathrm{~s}^{-1}$, the burning shell outside the core, from mass coordinate of $m=2.43 \mathrm{M}_{\odot}$ to $m=6.77 \mathrm{M}_{\odot}$, lives in the ashes of the previous oxygen-burning shell and is a violent silicon-burning shell with oxygen entrainment from the top, and the shell is in quasi-statistical equilibrium (QSE). At the bottom of the shell, the mass fraction of iron group elements made by silicon burning reaches $85 \%$ but drops to less than $1 \%$ at the top of the shell. In the outer region of the shell, clearly, the mixing time is longer than the burning time at the bottom. The high entropy (Figure 1, orange dashed line) may be due to the oxygen entrainment; most of the oxygen does not reach the bottom of the shell but burns at a mass coordinate of $\sim 4.5 \mathrm{M}_{\odot}$, reflected in a local maximum in specific energy generation rate. The actual oxygen burning shell starts at a mass coordinate of $m \approx 6.81 \mathrm{M}_{\odot}$, separated from the siliconburning shell by a semiconvective layer. It is still a quite powerful shell with a specific energy generation rate that is comparable to that of the silicon-burning shell, and it has a high specific entropy of $8.2 k_{\mathrm{B}} \mathrm{u}^{-1}$.

Concurrently with the collapse of the iron core, the entire outer part of the He core of $34.4 \mathrm{M}_{\odot}$ is already collapsing with velocities of several $100 \mathrm{~km} \mathrm{~s}^{-1}$ (Figure 2). In effect, the model experiences a combination of concurrent "classical" core collapse and pair instability in the oxygen-rich shells. The entire evolution of this model is shown in the Kippenhahn diagram in the left panel of Figure 3.

A peculiarity of the model is its close proximity to the pairinstability regime. As is not untypical for the transition between 

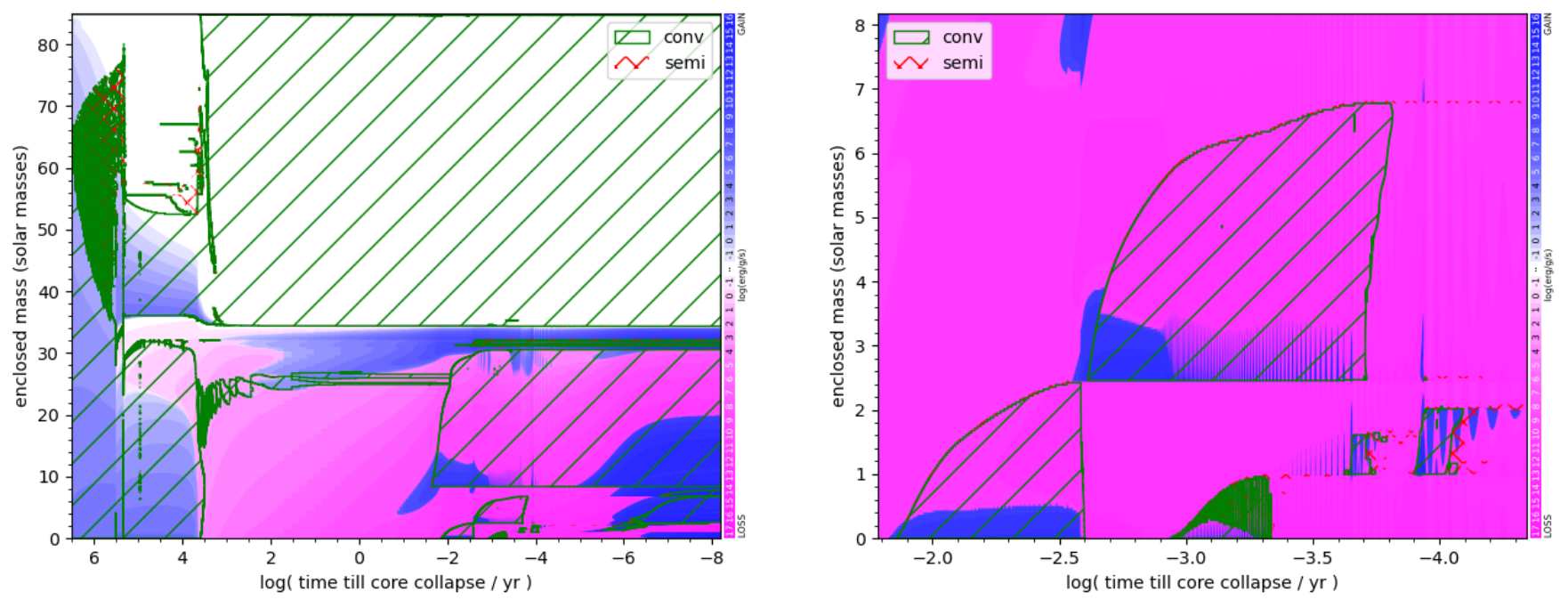

Figure 3. Kippenhahn diagrams for model $z 85$.

Left panel: The entire evolution from zero-age main sequence (ZAMS) to core collapse on a logarithmic time scale where we assume "core collapse" or core bounce, is reached $1 / 4$ second after the last model shown, which is a reasonable estimate. The $y$-axis shows the enclosed mass (mass coordinate). Green hatching indicates convective regions, also outlined by a green line; red cross hatching indicates semi-convective regions. The regions appearing solid green on the left side during core hydrogen burning around a mass coordinate of $60 \mathrm{M}_{\odot}$ is effectively semi-convective, but threaded though with many small convective zones that each contribute their green outlines to the plot. Blue shading indicates net specific nuclear energy generation rate, each level of increased shading intensity indicating an increase in energy generation rate by one order of magnitude, with the faintest level being $0.1 \mathrm{erg} \mathrm{g}^{-1} \mathrm{~s}^{-1}$. Purple shading indicates net specific nuclear energy loss, using the same scheme as for energy generation. For both, we plot the net value of energy generation and neutrino losses, as it is this that affects the evolution and structure of the star. Core hydrogen burning (main sequence, MS) is from the start until about 5.5 on the $x$-axis ( 300,000 yr prior to collapse). Below we adopt the short form " $x=5.5$." Core helium burning is until about $x=3.6$, the neutrino-powered CO core contraction phase is only $\sim 4,000 \mathrm{yr}$. There is a major core-envelope mixing event at around $x=5.4$ that leads to the establishment of a powerful convective hydrogen-burning shell between $36 \mathrm{M}_{\odot}$ and $53 \mathrm{M}_{\odot}$ that is enriched in CNO material from core helium burning, increasing entropy and preventing further core-envelope mixing. Core carbon burning starts in a radiative manner less than one year before collapse, neon burning is not prominent due to low abundances of carbon and neon made in this star. Core oxygen burning starts at around $x=-1.9,4$ days before core collapse, and core silicon burning starts at around $x=-3,8$ hours before core collapse. At the time of core oxygen ignition $(x=-1.9)$, an extended convective carbon-burning shell forms, reaching from $10 \mathrm{M}_{\odot}$ to $30 \mathrm{M}_{\odot}$ in mass coordinate and lasting until core collapse. At core helium depletion, the envelope undergoes another brief ( $2^{\text {nd }}$ ) dredge-up phase by about $2 \mathrm{M}_{\odot}$ and becomes a red supergiant with an extended convective envelope, from a mass coordinate of $34 \mathrm{M}_{\odot}$ (helium core size) to the surface, that lasts until core collapse.

Right panel A zoom-in of core and shell oxygen and silicon burning. We use the same $x$-axis as in the left panel. Starting just before $x=-3$ and at mass coordinate $3 \mathrm{M}_{\odot}$ we see many small vertical stripes in the oxygen-burning shell. These are due to an oscillatory instability occurring in this star so close to pair instability. These oscillations encompass the entire core, and are hence also seen in the core and shell silicon burning. In core silicon burning $(x=-3$ to $x=-3.3$ ) the oscillations also affect the convection, and, as before, the extended green regions are just the outlines of the many vertical convective zone boundaries. Due to the logarithmic nature of the $x$-axis the oscillations appear to become wider toward the right-hand side of the plot although the frequency remains about constant, just the dynamical time-scale of the core. A detailed frequency analysis is shown in Figure 4.

stable and unstable regimes (e.g., see Paczynski 1983; Heger et al. 2007 for the case of accreting neutron stars), in this case we observe an oscillatory instability in oxygen shell burning and beyond. In the right panel of Figure 3 these oscillations become visible as horizontal stripes in the energy generation and neutrino loss rates - both are very sensitive to temperature - during the late part of the first oxygen shell burning and in silicon core and shell burning. In fact, the oscillations may be present even at earlier times, however, the time step may have been too large to track them and the implicit hydro code would have smoothed them out. A dynamic spectrogram for the neutrino signal of the oscillations is shown in Figure 4. The oscillation starts (at least) $10 \mathrm{~h}$ before the collapse and has a frequency of about $2.2 \mathrm{mHz}$. As core collapse is reached, however, the global stability criterion

$\int_{0}^{M} \frac{P}{\rho}\left(\gamma_{\mathrm{ad}}-\frac{4}{3}\right) \mathrm{d} m>0$,

is violated and the inner $100,000 \mathrm{~km}$ undergo homologous collapse, superimposed with the homologous collapse of the iron core. In the above equation, $P$ is the pressure, $m$ is the mass coordinate, $M$ is the total mass of the star, and $\gamma_{\text {ad }}$ is the adiabatic index. The oscillations slowly dampen out in the last half hour of contraction to the final core collapse, and the oscillatory instability transitions to a runaway growing instability.

\subsection{Progenitor Model z100}

The $100 \mathrm{M}_{\odot}$ model has a distinctly different pre-collapse structure (Figure 1, blue lines). Compared to Model z85, it has less extreme values of core mass and explodability parameters, with an Fe core mass of $1.69 \mathrm{M}_{\odot}$, Ertl parameters $M_{4}=2.06 \mathrm{M}_{\odot}$, and $\mu_{4}=6.35$, and a compactness of $\xi_{2.5}=0.40$. The Ertl criterion still indicates $\mathrm{BH}$ formation, however. The shells outside the Fe core are nonconvective, and there are no entropy and density steps associated with convective shell interfaces.

The unusual progenitor structure - compared to normal CCSN progenitors - is due to the earlier evolution of the Model (Figure 5, left panel): About 1,500 years prior to collapse, the star experiences a sequence of four pair instability pulses of increasing strength and recurrence times (see Woosley 2017) within a few months. These pulses eject the entire hydrogen envelope, the helium layer, and the outer part of the $\mathrm{CO}$ core, leaving behind a $42.53 \mathrm{M}_{\odot}$ helium-free 


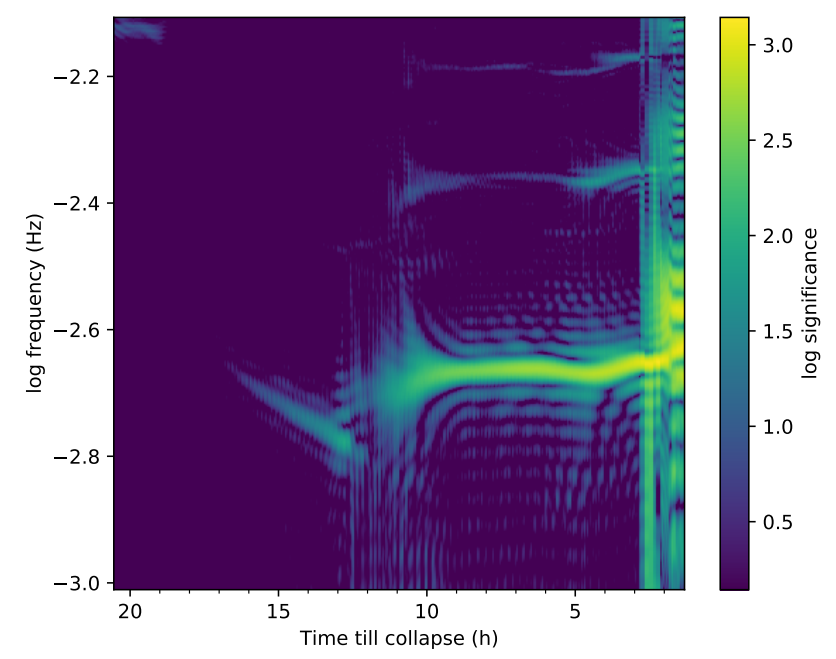

Figure 4. Dynamical power spectrogram of the last 20 hours of progenitor model z85 prior to collapse using a time window of $2^{13} \mathrm{~s}$. Details on the spectrogram code used are described in Tse et al. (2021). Here we plot the stellar neutrino luminosity of the star as this is an integral quantity over the entire star with a strong signal due to the high temperature-sensitivity of neutrino loss rates. The onset of the clear signal with a frequency of about $10^{-2.65} \mathrm{~Hz}$ (period of $450 \mathrm{~s}$ ) at $10 \mathrm{~h}$ before collapse corresponds to the clear oscillations seen starting in the Si shell at $3 \mathrm{M}_{\odot}$ and $0.001 \mathrm{yr}$ on the right-hand side of Figure 3. This pulsational instability may be present earlier than seen in this model calculation but the simulation time step may have been too large to trace it in earlier evolution phases.

core that is dominated by oxygen, and with only small mass fractions of neon $(8 \%)$, carbon (5\%), and magnesium (1\%). The pulses (Figure 5, right panel) lead to an increase of entropy and density (Figure 6) in the core, and "explosive" oxygen and silicon burning during the pulses - that actually powered the pulses - lead to depletion of oxygen and even silicon in the core. After the last pulse only a small amount of oxygen remained below $\sim 5.5 \mathrm{M}_{\odot}$, and in the centre a mass fraction of $66 \%$ of iron group elements was made and only a mass fraction of $27 \%$ of silicon and sulphur remained (plus $\sim 7 \%$ of calcium and argon). As a result, in the final pre-collapse evolution there is no convective oxygen burning, neither core nor shell, and the silicon core and shell burning are rather weak and not very extended.

It is this rather fast final evolution with runaway cooling and burning only in the centre, in the wake of the pair-instability pulse, that causes the rather high-entropy and low-density envelope: there is not enough time to lose entropy by neutrino emission after the pulse. On close inspection, the difference can be seen as much more intensive purple shades before the first pulse at $x=-4.9$, in the right panel of Figure 5, as compared to the final distribution of purple shading in the core before collapse at $x=-8.2$ in the left panel of Figure 5 .

\section{SUPERNOVA SIMULATIONS - NUMERICAL METHODS AND SETUP}

The simulations in this study are performed using the neutrino hydrodynamics code CoCoNuT-FMT. The setup of our 3D simulations is similar to our previous studies (Powell \& Müller 2019, 2020) with the exception of the EoS, however we repeat some of the details here for completeness.

We use a general relativistic finite-volume solver for the equa- tions of hydrodynamics (Dimmelmeier et al. 2002; Müller et al. 2010, 2019) formulated in spherical polar coordinates and the fast multi-group transport (FMT) method of Müller \& Janka (2015) for the neutrino transport. The GW emission is extracted by the time-integrated quadrupole formula (Finn 1989; Finn \& Evans 1990; Blanchet et al. 1990) with relativistic correction factors as derived in Müller et al. (2013). The simulations are run with a spatial resolution of $550 \times 128 \times 256$ zones in radius, latitude, and longitude. We employ a non-equidistant radial grid that reaches out to a radius of $10^{5} \mathrm{~km}$.

We use three different EoS at high densities that all match well with recent neutron star observations, namely the Lattimer \& Swesty EoS with a bulk incompressibility of $\mathrm{K}=220 \mathrm{MeV}$ (LS220; Lattimer $\&$ Swesty 1991), and the SFHo and SFHx EoS from Steiner et al. (2013). For cold matter in $\beta$-equilibrium, the radius of a $1.4 \mathrm{M}_{\odot}$ neutron star is $11.88 \mathrm{~km}$ for SFHo, $11.97 \mathrm{~km}$ for SFHx, and $12.62 \mathrm{~km}$ for LS220. The maximum neutron star mass is $2.059 \mathrm{M}_{\odot}$ for SFHo, $2.130 \mathrm{M}_{\odot}$ for SFHx, and $2.06 \mathrm{M}_{\odot}$ for LS220. These values are consistent with the latest constraints from $\mathrm{GW}$ observations (Abbott et al. 2018; Capano et al. 2020), and pulsar and X-ray surveys (Landry et al. 2020; Raaijmakers et al. 2020). The SFHo and SFHx EoS are consistent with the latest nuclear constraints, however LS220 is incompatible with known nuclear constraints (Tews et al. 2017). It should be pointed out, however, that compliance with constraints on cold neutron stars, the nuclear incompressibility, and the nuclear symmetry energy and its derivative does not necessarily guarantee (superior) accuracy in the supernova problem because of finite temperatures. Other parameters such as the nucleon effective mass can become critical (Yasin et al. 2020); and arguments can be made that tuning the parameters of Skyrme-type or meson-exchange models to nuclear properties at saturation densities is not sufficient to ensure correct behaviour in the supernova regime (Furusawa et al. 2017). Given the remaining uncertainties about the EoS in the regime relevant to supernovae, an exploration of different models remains useful. At low density, we use an EoS accounting for photons, electrons, positrons, and an ideal gas of nuclei together with a flashing treatment for nuclear reactions (Rampp \& Janka 2002).

In total, five different models have been simulated. Model z85 has been simulated with all three EoS, and model z100 has been simulated using the SFHx and SFHo EoS only. The models are labelled as PROGENITOR_EoS (see Table 1).

\section{EXPLOSION MODEL DYNAMICS}

In this section, we discuss the dynamical evolution and, where applicable, the explosion and remnant properties of our models. The outcomes of the five simulations are summarised in Table 1. The average shock radii for all models are shown in Figure 7 (top left panel). The three $\mathrm{z} 85$ models all undergo shock revival before $\mathrm{BH}$ formation, whereas the shock still has not started to move out in the z 100 models. In some respects, the behaviour of the two progenitors corresponds to trends found by Ott et al. (2018) in that the z85 models with a very massive low-entropy core and high post-bounce mass accretion rates (Figure 7, top right panel) explode more readily than the $z 100$ models with lower post-bounce accretion rates. A close examination of the two sets of models reveals important differences to the findings of Ott et al. (2018), however. 

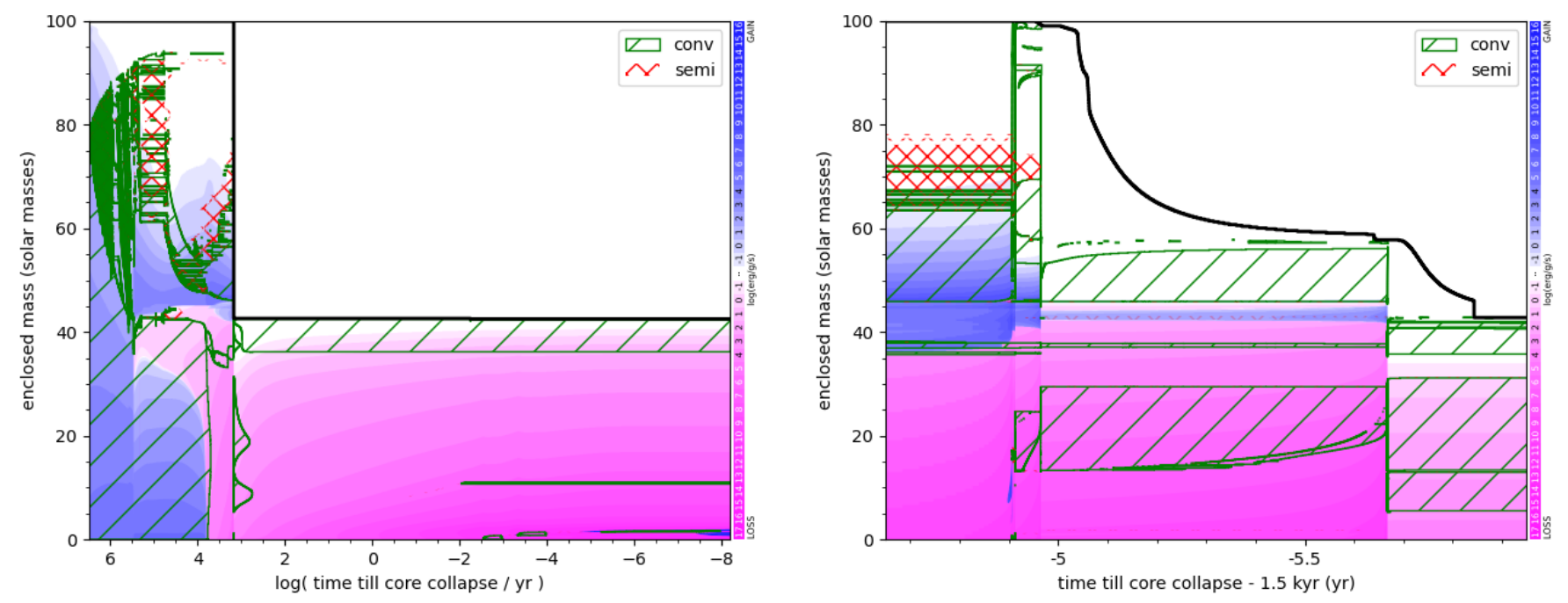

Figure 5. Kippenhahn diagrams for the evolution of model z100.

Left panel: The entire evolution from ZAMS to core collapse similar to Figure 3. Core hydrogen burning ends at about $x=5.5$ and core helium burning ends at $x \approx 3.8$. At $1.5 \mathrm{kyr}$ prior to final collapse $(\mathrm{x}=3.176$ ) the star undergoes a sequence of four pair-instability pulses (right panel, discussed below) that lead to the ejection of the hydrogen envelope, the helium shell, and the outer fringes of the CO core such that an oxygen-dominated core of only $42.53 \mathrm{M}_{\odot}$ remains at the time of core collapse. The outer convection zone seen here is in that CO core with a carbon mass fraction of only $5 \%$. In the final post-pulse evolution, core silicon burning occurs from $x=-2.5$ to $x=-3$ and two silicon-burning shells from $x=-3.2$ to $x=-4$ and $x=-5.2$ to $x=-8$. Core neon and oxygen have already been depleted in powering the pair-instability pulses.

Right panel: At $\sim 1,000 \mathrm{yr}$ before the final core collapse, the star encounters radiative core and shell carbon burning and the pair instability sets in, leading to rapid contraction that is eventually stopped by "explosive" (rapid radiative) core neon and oxygen burning at $x=-4.9$ ( $1,495.1 \mathrm{yr}$ prior to final core collapse). Due to the pulse contraction only taking minutes, it is not clearly visible at the scale shown here for the purpose of providing an overview. This is followed by a sequence of three further pulses on a recurrence time-scale of days to months. See Figure 6 for details of the core density evolution. Each of the pulses leads to rapid burning - seen as shells rapidly burning outward in the lead-up to the pulse - increasing entropy in the core and thereby reducing post-pulse density and temperature, visibly leading to a reduction in specific neutrino loss rates. The star has to cool on the Kelvin Helmholtz time scale for the next pulse. For the first pulses the cooling is clearly powered by neutrino losses, but after the last pulse neutrino losses become inefficient at first (see also Woosley 2017), leading to a longer recovery time to the final collapse with a quite altered core structure. During the pulses, neon and oxygen are depleted in the core such that the usual hydrostatic convective neon and oxygen burning core and shell phases years to weeks prior to collapse as seen, e.g., for model z85 (Figure 3; Woosley et al. 2002) cannot occur.

\begin{tabular}{lllccccc}
\hline Model & Progenitor & EoS & $\begin{array}{c}t_{\text {sh }} \\
(\mathrm{s})\end{array}$ & $\begin{array}{c}E_{\text {diag }} \\
\left(10^{51} \mathrm{erg}\right)\end{array}$ & $\begin{array}{c}M_{\text {rem }} \\
\left(\mathrm{M}_{\odot}\right)\end{array}$ & $\begin{array}{c}t_{\mathrm{BH}} \\
(\mathrm{s})\end{array}$ & $\begin{array}{c}R_{\text {Shock }} \\
(\mathrm{km})\end{array}$ \\
\hline z85_SFHx & $\mathrm{z} 85$ & SFHx & 0.298 & 2.7 & 2.57 & 0.59 & 4,451 \\
\hline z85_SFHo & $\mathrm{z} 85$ & SFHo & 0.207 & 1.25 & 2.44 & 0.36 & 2,103 \\
\hline z85_LS220 & \multirow{2}{*}{$\mathrm{z85}$} & LS220 & 0.160 & 0.7 & 2.51 & 0.29 & 1,504 \\
\hline z100_SFHx & \multirow{2}{*}{$\mathrm{z} 100$} & SFHx & - & - & 1.88 & $\gg 0.5$ & 89 \\
\hline z100_SFHo & $\mathrm{z} 100$ & SFHo & - & - & 2.05 & $\gg 0.5$ & 60 \\
\hline
\end{tabular}

Table 1. Summary of model setup and outcomes. $t_{\mathrm{sh}}$ is the time of shock revival, $E_{\mathrm{diag}}$ is the diagnostic energy at the end of the simulation, $M_{\mathrm{rem}}$ is the mass of remnant at the end of the simulation, $t_{\mathrm{BH}}$ is the time after bounce of black hole formation, and $R_{\text {shock }}$ is the shock radius at the end of the simulation. The SFHx EoS, which supports the highest maximum mass, is the least favourable to explosion but produces a larger diagnostic energy due to the longer accretion time.

\subsection{Progenitor Model z85}

In the $z 85$ explosion models, shock revival occurs early after bounce with the average shock radius crossing $300 \mathrm{~km}$ at $0.160 \mathrm{~s}$ (LS220) $0.207 \mathrm{~s}$ (SFHo), and $0.298 \mathrm{~s}$ (SFHx), respectively (Table 1). The ratio between the advection and heating time scale $\tau_{\text {adv }}$ and $\tau_{\text {heat }}$, which quantifies the proximity to runaway shock expansion (Janka 2001; Buras et al. 2006; Müller 2020), exceeds the critical threshold $\tau_{\text {adv }} / \tau_{\text {heat }}=1$ even earlier at times of $0.128 \mathrm{~s}$ (LS220), $0.158 \mathrm{~s}$ (SFHo), and $0.257 \mathrm{~s}$ (SFHx) (Figure 8) when the shock is still within the low-entropy core as can be seen from the PNS masses at the corresponding times (Figure 7, bottom left). It is difficult to unambiguously associate the different shock trajectories and proto-neutron star radii (Figure 7, bottom right) with the microphysical properties of the different EoS. For EoS that differ more markedly the impact of the microphysics on the heating conditions is now better understood; for example Yasin et al. (2020) recently identified the low effective nucleon mass as the critical factor that explains adverse heating conditions in case of the Shen EoS (Shen et al. 1998). While this may play a role in explaining the different explosion times of the $\mathrm{z} 85$ models since the SFHo and SFHx EoS also have lower ef- 


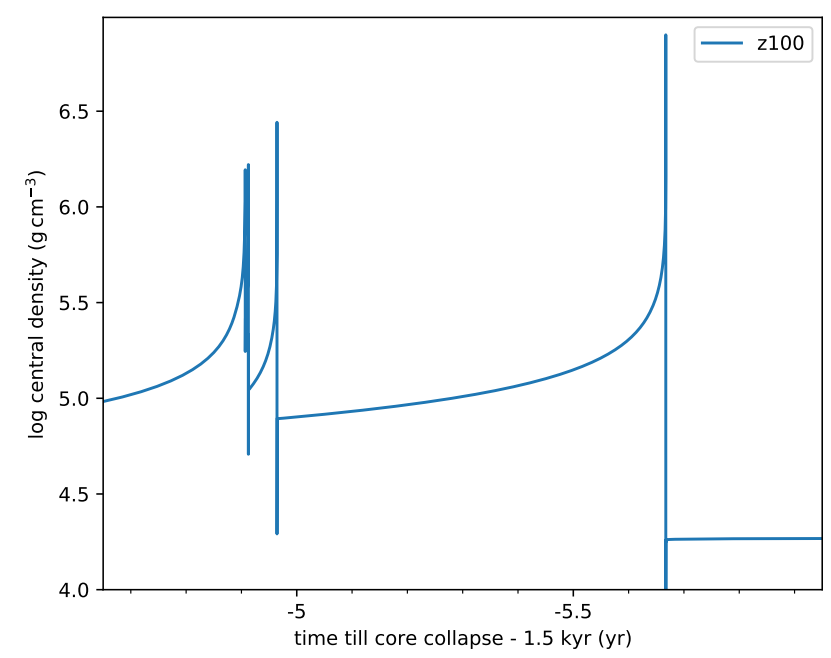

Figure 6. Central density as a function of time of model z100 for the same time range as in the right panel of Figure 5. Shown are all four pulses of pulsational pair instability supernova (PPSN) the model encounters, $1.5 \mathrm{kyr}$ before the final collapse. The first two pulses are only 2 days apart, the third pulse occurs 19 days after the second, and the final fourth pulse occurs 145 days after the third pulse. The pulses become increasingly more powerful, leading to lower post-pulse density with accordingly longer wait times for the next pulse as well as large mass ejections. The maximum density peaks during the pulse $(\sim 30 \mathrm{~s})$ as well as the post-pulse ring-down (oscillation period is of a fraction of an hour and a comparable decay timescale) are not resolved in this plot and are visible as vertical lines only. They are not relevant for the discussion of the presupernova models. For more details on PPSNe see Woosley (2017).

fective nucleon masses than LS220 (Steiner et al. 2013), there are confounding factors that might influence the order of shock revival. The models with different EoS exhibit differences in mass accretion rate and PNS mass already early on. This is due to the interplay of different collapse times for the three EoS and the peculiar evolution of the progenitor towards collapse with a pair instability pulse that coincides with core collapse. An influence of minute EoS differences on the collapse time and early accretion history has been noted before (see Hüdepohl 2014, Section 3.2), and is ideally avoided by using the same low/intermediate-density EoS of different simulations up to densities of $\sim 10^{11} \mathrm{~g} \mathrm{~cm}^{-3}$ (Hüdepohl 2014; Bruenn et al. 2020). It is also worth pointing out that $\tau_{\text {adv }} / \tau_{\text {heat }}$ already reaches a value of 0.75 in model z85_SFHx before $0.2 \mathrm{~s}$ after bounce and only narrowly fails to explode earlier. The delay in shock revival compared to z85_LS220 and z85_SFHo may thus give an exaggerated difference between intrinsic EoS differences. It remains to be determined how the robust the hierarchy of shock revival times between LS220, SFHo, and SHFx is, but we do note that the order of shock revival between the LS220, SFHo, and SFHx models is consistent with other recent studies (Bollig et al. 2020; Landfield 2018),

All three models form BHs soon after shock expansion sets in. $\mathrm{BH}$ formation occurs at $0.59 \mathrm{~s}, 0.36 \mathrm{~s}$ and $0.29 \mathrm{~s}$ after core bounce for the SFHx, SFHo and LS220 models, respectively. Interestingly, even though model z85_SFHx takes the longest time to reach shock revival, the shock has propagated further than in the other two models by the time of $\mathrm{BH}$ collapse ${ }^{2}$ At least qualitatively, the differences in

2 We define the $\mathrm{BH}$ formation time as the point where the central density
$\mathrm{BH}$ formation time can be more easily explained than the differences in explosion time. After about $250 \mathrm{~ms}$ the PNS masses have become quite similar and the different maximum mass of warm neutron stars becomes the most important factor (cf. Steiner et al. 2013; da Silva Schneider et al. 2020) that results in z85_SFHx forming a BH later than z85_SFHo and z85_LS220. The mass accreted onto the PNS is also higher for z85_SFHx $\left(0.114 \mathrm{M}_{\odot}\right)$ than for z85_SFHx $\left(0.100 \mathrm{M}_{\odot}\right)$ and z85_LS220 $\left(0.085 \mathrm{M}_{\odot}\right)$.

Model z85_SFHx also has the highest diagnostic explosion energy at the time of $\mathrm{BH}$ collapse, with a value of $2.7 \times 10^{51} \mathrm{erg}$ as opposed to $1.25 \times 10^{51} \mathrm{erg}$ for $\mathrm{z} 85$ _SFHo and $\mathrm{z} 85$ _LS220. This is due to the longer accretion time before the maximum PNS mass is reached. The energetics and ultimate fate (i.e., whether the shock manages to propagate outward and expel the envelope) of supernovae with early fallback could therefore prove very sensitive to the nuclear EoS.

To determine the final fate of the "aborted" explosion in the three $85 \mathrm{M}_{\odot}$ models, long-time simulations in the vein of Chan et al. (2018) would be required. Despite progress on the theory of mass ejection by weak explosions (Chan et al. 2020; Mandel \& Müller 2020; Matzner \& Ro 2020; Linial et al. 2020), several scenarios are conceivable. In all cases, the binding energy of the shells outside the shock by far exceeds the diagnostic explosion energy at the time of BH collapse; even for model z85_SFHx, this "overburden" is still $5-6 \times 10^{51} \mathrm{erg}$. Since, however, the pre-shock infall velocities are already subsonic in model z85_SFHx, it is likely that the shock will continue to propagate outwards for a substantial time and transition to the weak-shock regime as it scoops up bound pre-shock material (Chan et al. 2018, 2020). It has been argued (Mandel \& Müller 2020; Matzner \& Ro 2020; Linial et al. 2020) that the energy or acoustic luminosity of the resulting sound pulse is approximately conserved and then determines the amount of material ejected from the surface. Given the large ratio of envelope binding energy to diagnostic explosion energy it is still a distinct possibility that the shock will not reach the surface.

For model z85_LS220, the situation is different in that the shock has just barely reached the sonic point of the infall region at the time of BH collapse, and the shock is already weaker to begin with. It therefore appears likely that the shock cannot escape the newly formed $\mathrm{BH}$ since a weak sound pulse would be too slow to propagate outward through the infalling pre-shock matter.

Despite these uncertainties, we can obtain a conservative lower limit for the final $\mathrm{BH}$ masses of our models. Using the (extremely optimistic) assumption that the sound pulse carries the initial explosion energy without any loses, we can match this energy with the binding energy of the ejected shells and thereby estimate the minimum final BH masses of $30.7 \mathrm{M}_{\odot}$ for z85_SFHx, 32.4 $\mathrm{M}_{\odot}$ for z85_SFHo, and $33.2 \mathrm{M}_{\odot}$ for z85_LS220. In case the hydrogen envelope of the progenitor has been lost due to binary interaction, we would predict a fairly narrow range of $30.7-34.4 \mathrm{M}_{\odot}$ for the $\mathrm{BH}$ mass.

\subsection{Progenitor Model z100}

The two $z 100$ explosion models do not achieve shock revival before the end of the simulation time. The time-scale criterion $\tau_{\text {adv }} / \tau_{\text {heat }}$ has

exceeds the boundaries of the EoS table. The boundary is encountered slightly earlier for the SFHx and SFHo EoS than for LS220. Since BH formation generally occurs between the $2 \mathrm{~ms}$ output intervals, there is usually no output file available exactly at this point in time. In the last output file, the central density and lapse have typically reached values of $\rho_{\mathrm{c}} \gtrsim 10^{15} \mathrm{~g} \mathrm{~cm}^{-3}$ and $\alpha_{\mathrm{c}}=0.4-0.45$, respectively. 

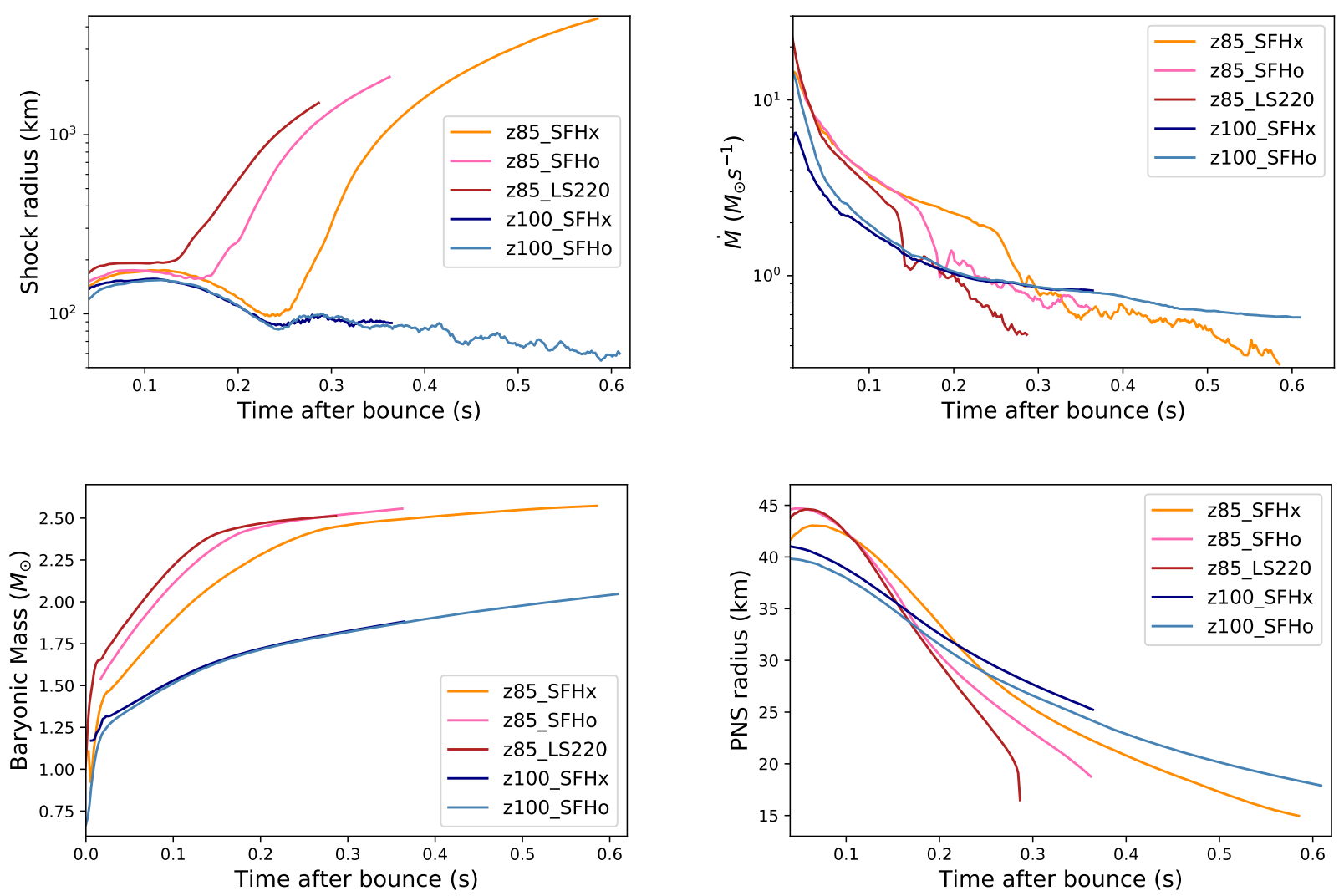

Figure 7. Angle-averaged shock radii (top left), mass accretion rate at $200 \mathrm{~km}$ (top right) baryonic PNS mass (bottom left), and PNS radius (bottom right) for all models. The z85 models achieve shock revival early after core bounce and then form black holes within hundreds of milliseconds. The z100 models have a high mass accretion rate but do not reach black hole formation before the end of the simulation.

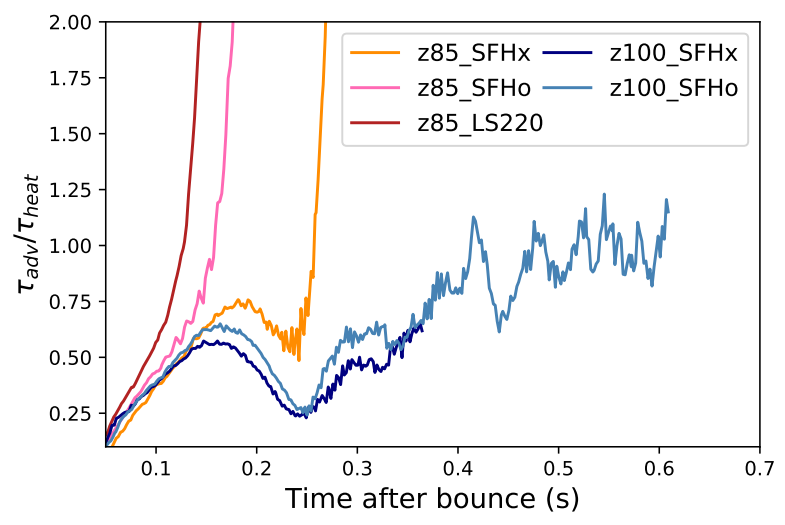

Figure 8. The time-scale criterion $\tau_{\text {adv }} / \tau_{\text {heat }}$ for all models. The time-scale criterion indicates conditions for neutrino-driven runaway shock expansion at $\tau_{\text {adv }} / \tau_{\text {heat }} \gtrsim 1$. The time-scale criterion values for the $z 100$ models indicate that the shock would likely be revived for these models some time after $0.6 \mathrm{~s}$.

a clear upward trend for these two models, however, and has already reached the critical value $\tau_{\text {adv }} / \tau_{\text {heat }}=1$ at $0.41 \mathrm{~s}$ for $\mathrm{z} 100 \_\mathrm{SFHx}$ (Figure 8). It is therefore likely that the shock would be revived in these two models some time after $0.6 \mathrm{~s}$. Even though the mass accretion rate is still quite high in the $z 100$ model at the end of the simulations, the baryonic PNS masses are still quite far away from the maximum values allowed for their respective EoS. The most probable outcome for these models is therefore that they will experience shock revival, but still undergo delayed collapse to a $\mathrm{BH}$. Since the binding of the shells ahead of the shock is $\gtrsim 4 \times 10^{51} \mathrm{erg}$, it is unlikely that a neutrino-driven explosion could still become strong enough to completely expel the envelope. As we could not follow these two models into the explosion phase and up to $\mathrm{BH}$ formation, we cannot assess whether there is any chance of partial mass ejection, or whether the entire metal core left by the previous pair instability pulse will completely collapse to a $\mathrm{BH}$.

\subsection{EoS dependence of SASI activity}

We find that the EoS qualitatively affects the nature of the hydrodynamic instabilities during the pre-explosion phase. To diagnose SASI activity in our models, we decompose the angle-dependent shock position $r_{\mathrm{sh}}(\theta, \varphi)$ into spherical harmonics $a_{l m}$,

$a_{l m}=\frac{(-1)^{m}}{\sqrt{4 \pi(2 l+1)}} \int Y_{l m}^{*}(\theta, \varphi) r_{\mathrm{sh}}(\theta, \varphi) \mathrm{d} \Omega$,

where $Y_{l m}$ are real spherical harmonics with the same normalisation as in Burrows et al. (2012). In Figure 9, we show the normalised dipole and quadrupole coefficients of the shock position. We also illustrate the multi-dimensional structure of the flow in models z85_SFHo and z100_SFHo at selected epochs using 2D slices of the entropy in the supernova core in Figure 10. 


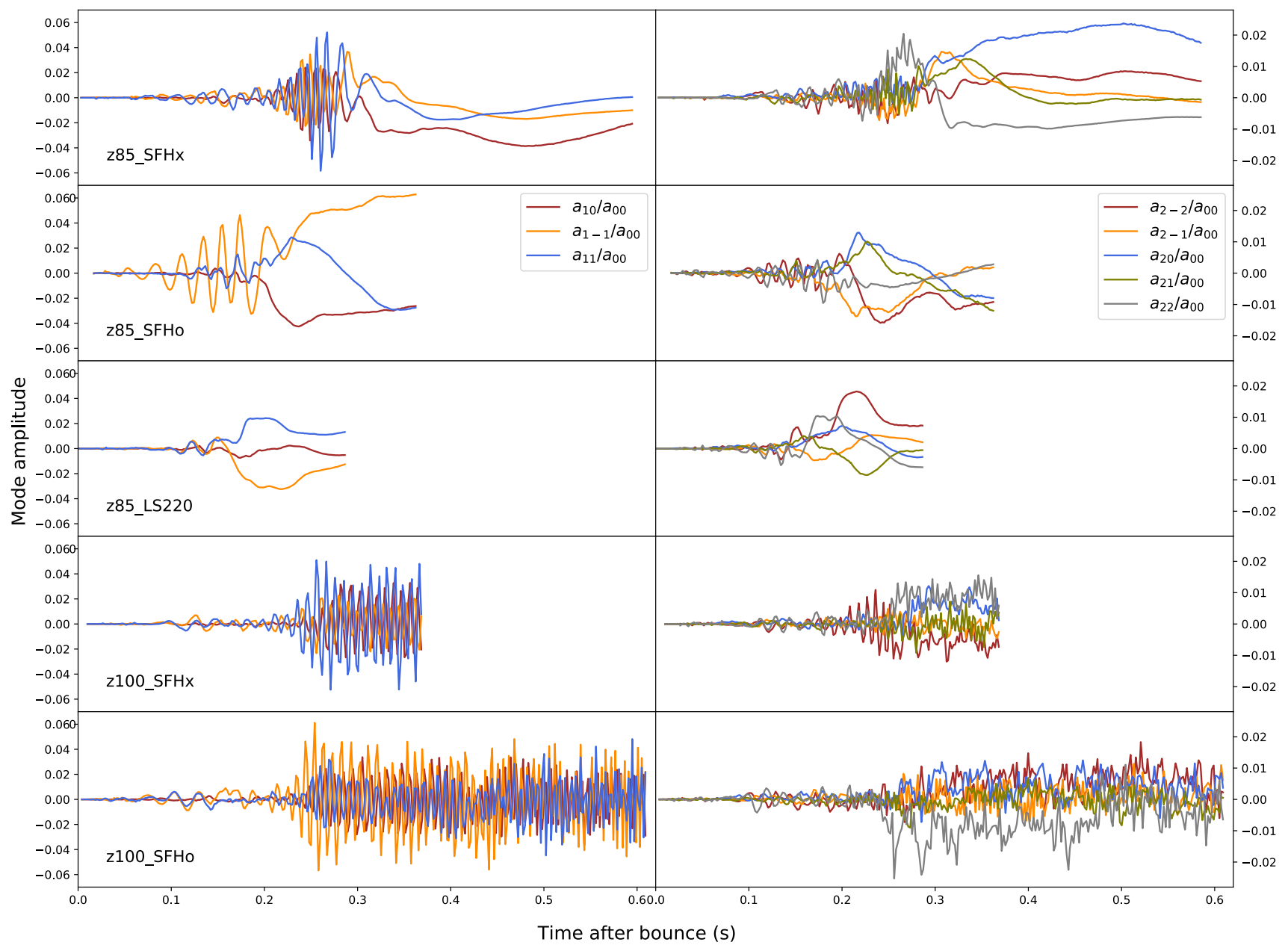

Figure 9. Normalised dipole $\left(a_{1 m} / a_{00}\right)$ and quadrupole coefficients $\left(a_{2 m} / a_{00}\right)$ of the angle-dependent shock position.

All of the SFHo and SFHx models develop strong SASI activity at some point, whereas model z85_LS220 hardly develops quasiperiodic shock oscillations and is clearly convectively dominated around shock revival. A trend towards strong SASI activity with the SFHx EoS was already found by Kuroda et al. (2016). It is noteworthy that strong SASI also occurs in the exploding models z85_SFHx and z85_SFHo in contrast to the findings of Ott et al. (2018), who posited that rapidly developing explosions in progenitors with high compactness are dominated by convection from the outset. Models z85_SFHx and z85_SFHo rather develop SASI activity earlier than the $z 100$ models with lower accretion rates. The $z 100$ models rather go through a regime where rather weak SASI activity and convective plumes can be seen side by side (Figure 10, top left) before developing stronger and cleaner SASI oscillation later from about $0.25 \mathrm{~s}$ onward (Figure 10, top right). The SASI then maintains strong and stable dipole modes (for several hundred milliseconds in z100_SFHo).

In all models with SASI activity, the dipole mode appears to be dominant. The models do not show pronounced quasi-periodic oscillations in the quadrupole coefficient $a_{2 m}$ most of the time. There are, however, hints of modest quasi-periodic quadrupolar oscillations in model z85_SFHo between $0.1 \mathrm{~s}$ and $0.2 \mathrm{~s}$ and in $\mathrm{z} 100 \_\mathrm{SFHx}$ between $0.2 \mathrm{~s}$ and $0.25 \mathrm{~s}$. Similar to the BH-forming models of Walk et al. (2020), a pronounced SASI quadrupole only appears episod- ically, and different from the models of Walk et al. (2020). This does not, of course, not argue against the existence of a regime with a dominant quadrupole mode in some $\mathrm{BH}$-forming progenitors; a dominant quadrupole simply does not emerge for the two particular progenitors considered in this study, and the emergence of a dominant quadrupole may hinge on details of the neutrino transport and EoS effects like muonisation (Bollig et al. 2017) that are not included in our models.

\section{GRAVITATIONAL WAVES}

\subsection{Features of the GW signal}

The time series and spectrograms of the GW emission of our models are shown in Figure 11 for a selected observer direction in the equatorial plane of the spherical polar grid. All of the models show the typical g-mode emission which rises in frequency with time from a few hundred $\mathrm{Hz}$ to $\sim 900 \mathrm{~Hz}$. The effects of the different EoS are imprinted on the g-mode GW signals. As the g-mode GW frequency is $\propto M_{\mathrm{PNS}} / R_{\mathrm{PNS}}^{2}$ (Müller et al. 2013), the different EoS change how quickly the g-mode frequency rises in time. The rise will be dictated by the effective warm mass-radius relation $\mathrm{R}(\mathrm{M})$ (defined by a density of $10^{11}-10^{12} \mathrm{~g} \mathrm{~cm}^{-3}$ examples of which are shown, e.g., in Figure 3 

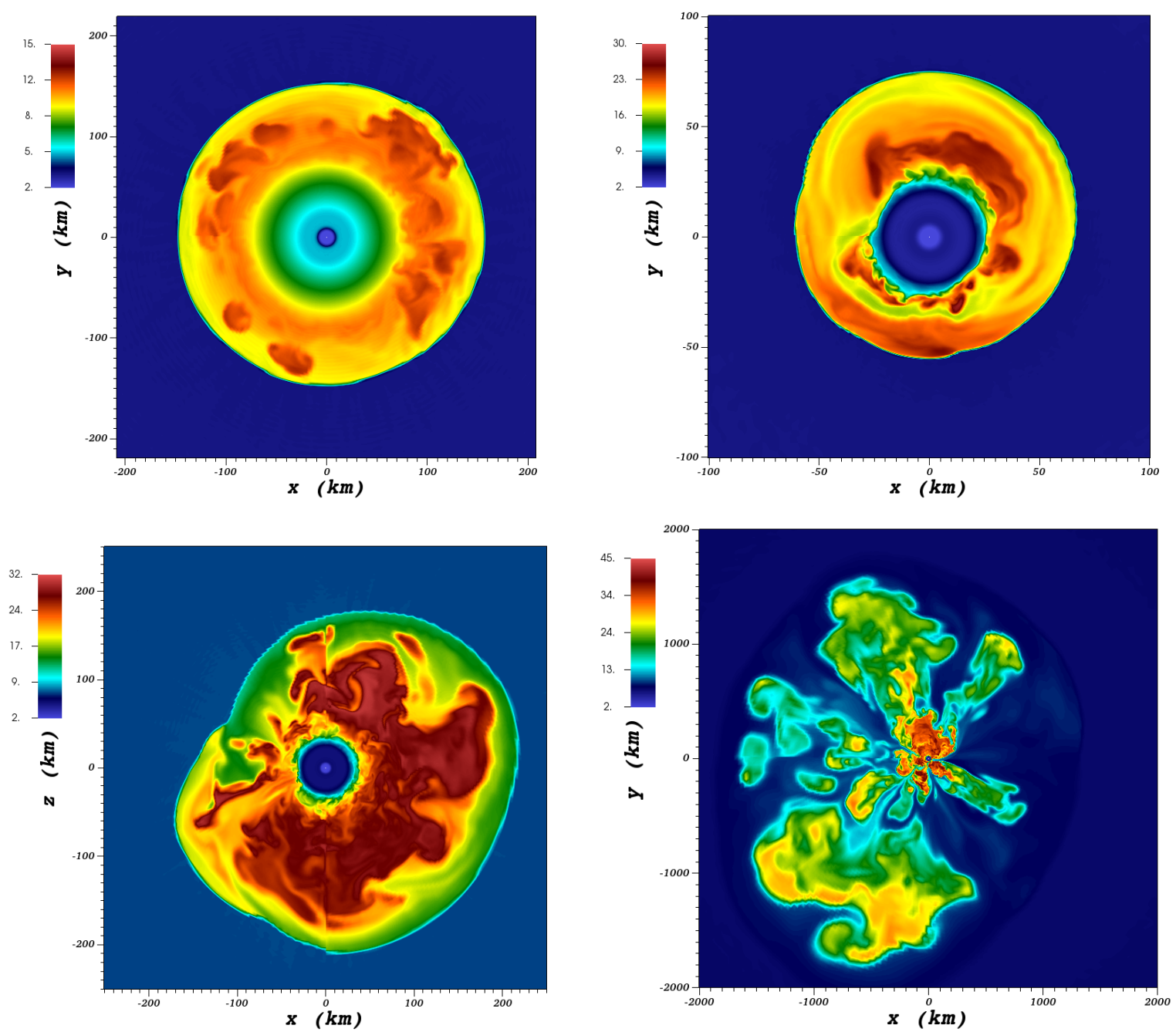

Figure 10. 2D slice of the entropy in model z100_SFHo at $100 \mathrm{~ms}$ and $450 \mathrm{~ms}$ post-bounce (top) and at $280 \mathrm{~ms}$ and $450 \mathrm{~ms}$ in model z85_SFHx. All the SFHo and SFHx models develop strong SASI activity. In the z100 models, SASI starts out as weak with some Rayleigh-Taylor convection on top (top left panel) and then becomes stronger with a more distinct SASI flow morphology later on (top right). In the z85 models, strong SASI develops early on (bottom left) and plays a crucial role in expanding the shock radius until neutrino heating conditions become critical. The SASI then freezes out and buoyancy-driven outflows develop in the explosion phase (bottom right).

of Sotani et al. 2017), which is related to the properties of the EoS and the time dependence of $M(t)$. The time-dependence of $M(t)$ could be reconstructed from the neutrino signal (Müller \& Janka 2014). One should note, however, that the warm mass-radius relation does depend on the entropy profiles and is somewhat progenitor- and time-dependent. Precision measurements of EoS properties through the g-mode frequency are therefore not realistic. The GW frequency increases more rapidly for LS220 than for SFHo or the SFHx model which has the slowest rise in GW frequency with time. In the $z 85$ models, the GW power from this mode peaks shortly after shock revival, which is also affected by the different EoS. As a consequence, the frequency around peak emission (which will mostly determine the overall spectrum) is lower in models that explode faster, in our case $\sim 650 \mathrm{~Hz}$ for LS220, $\sim 695 \mathrm{~Hz}$ for SFHo, and $\sim 710 \mathrm{~Hz}$ for SFHx. The $z 100$ models, which do not achieve shock revival, reach their maximum GW amplitudes between $0.25 \mathrm{~s}$ and $\sim 0.3 \mathrm{~s}$ once the shock has contracted sufficiently for strong SASI to set in. As the shock contracts further, the mass in the gain region decreases, the SASI motions that excite the g-mode carry less energy, again resulting in smaller GW amplitudes. Peak GW emission from the g-mode occurs at frequencies of $\sim 700 \mathrm{~Hz}$ for z100_SFHx and $\sim 695 \mathrm{~Hz}$ for z100_SFHo.

The g-mode emission of all our models is of lower frequency than the results obtained in some recent work by other groups which still have high GW amplitudes at frequencies over 1, $000 \mathrm{~Hz}$ (O'Connor \& Couch 2018; Radice et al. 2019; Mezzacappa et al. 2020; Pan et al. 2020). This means that our models are in a better frequency band for current ground-based GW detectors, and those from other groups may be more promising sources for proposed future high frequency GW detectors (Ackley et al. 2020). It is also noteworthy that BHforming models will not generically be distinguished by particularly high GW frequencies if the bulk of the GW power comes from a phase when the g-mode frequency is still low.

The relation between between the mass and radius of the PNS and the mode frequency is largely consistent with semi-analytic estimates (Müller et al. 2013) as in our previous non-rotating models (Powell \& Müller 2019, 2020) and the universal relations in Torres-Forné et al. (2019), especially during the pre-explosion phase. A comparison of 

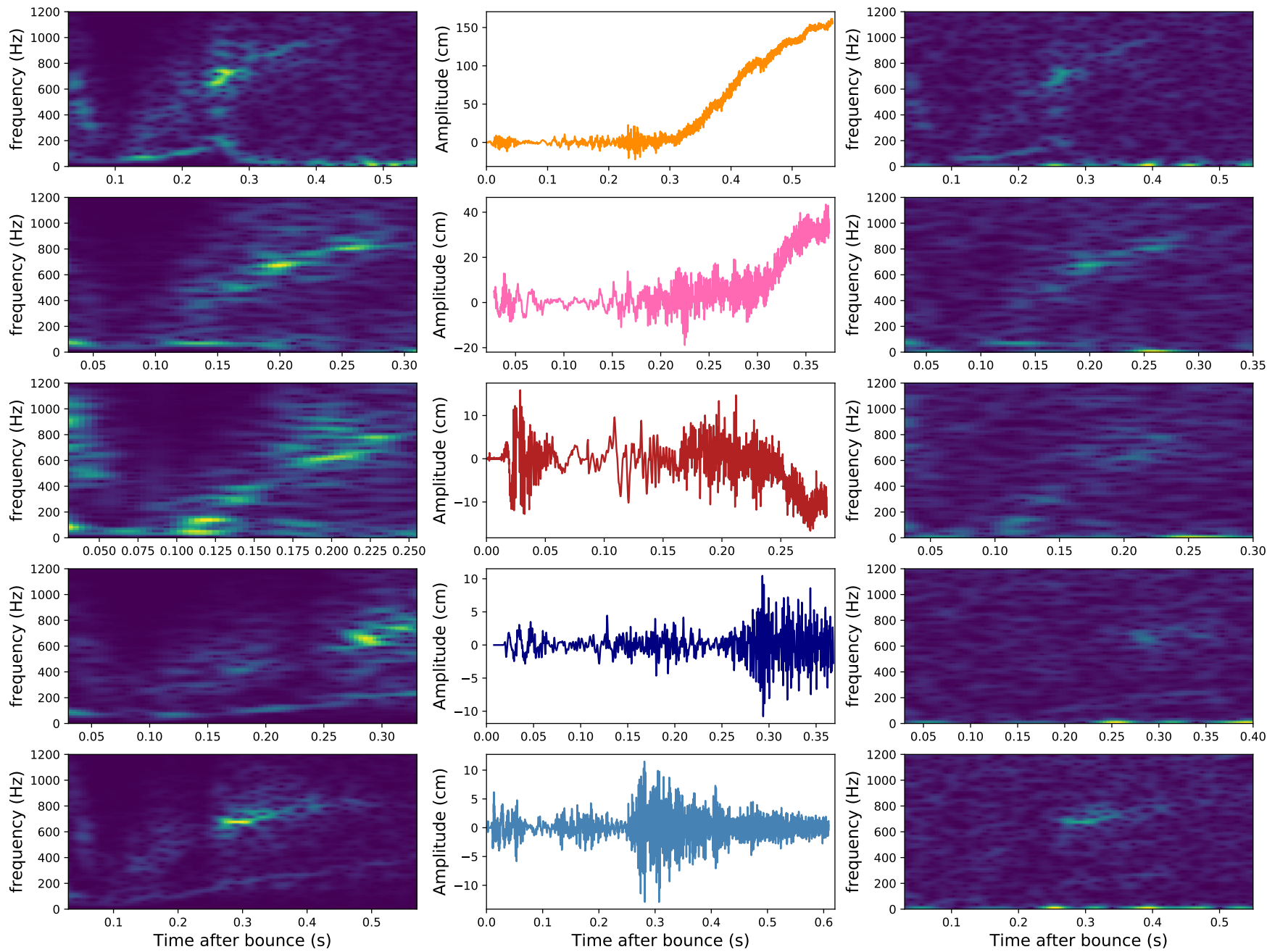

Figure 11. Gravitational wave signals and spectrograms for models z85_SFHx, z85_SFHo, z85_LS220, z100_SFHx, and z100_SFHo (top to bottom). The middle column shows the amplitude $h_{+}$of the plus polarisation mode for an observer in the equatorial plane at $(\theta, \varphi)=\left(90^{\circ}, 90^{\circ}\right)$. The left and right columns show the corresponding spectrograms without noise (left) and with Gaussian design sensitivity aLIGO noise at a distance of 5 kpc (right).

the spectrogram of model z85_SFHx and model z100_SFHx with the frequency relation for the ${ }^{2} g_{2}$ mode from Torres-Forné et al. (2019) is shown in a separate Figure 12 for improved clarity. We show these two models as an example but find the same results for all models. In fitting the dominant emission frequency one has to bear in mind that the emitting mode can change character to an f-mode (Morozova et al. 2018; Sotani \& Takiwaki 2020), but in practice the suggested scaling of the ${ }^{2} g_{2}$ frequency with $M / R^{2}$ also gives a reasonable fit with the f-mode frequency after the character of the mode changes. During the explosion phase the actual mode frequency from the spectrograms increases more slowly than the analytic scaling relations suggest, which has also been observed previously in Müller et al. (2013). These deviations from the analytic scaling relations in the explosion phase contribute to the dominance of relatively low frequencies in the overall signal in the $\mathrm{z} 85$ models despite the strong contraction of the PNS on the way to $\mathrm{BH}$ formation.

All of our models clearly show low-frequency GW emission at $\lesssim 200 \mathrm{~Hz}$ as well. The effects of the different EoS are more significant in the low-frequency GW emission. In the case of model z85_LS220, where the shock is revived very early, the low-frequency emission is quite strong, but rather spread out in frequency. It reflects irregular mass motions in the gain region with characteristic time scales of order $O(10 \mathrm{~ms})$ rather than periodic SASI oscillations. There may also be some confusion between genuine low-frequency emission from mass motions in the gain region and g-mode emission early on around $100 \mathrm{~ms}$, when the g-mode frequency is still very low.

In the SFHo and SFHx models, the low-frequency GW emission can clearly be attributed to the SASI. The low-frequency emission occurs in a rather clearly defined band in the spectrograms. The SASI frequency can be approximated as

$f_{\mathrm{SASI}}=\frac{1}{19 \mathrm{~ms}}\left(\frac{R_{\mathrm{sh}}}{100 \mathrm{~km}}\right)^{-3 / 2}\left[\ln \left(\frac{R_{\mathrm{sh}}}{R_{\mathrm{PNS}}}\right)\right]^{-1}$,

where $R_{\mathrm{sh}}$ is the shock radius and $R_{\mathrm{PNS}}$ is the radius of the PNS (Müller \& Janka 2014). As previously noted by Andresen et al. (2017), the SASI emission band in the GW spectograms is located at $2 f_{\text {SASI }}$ because of frequency doubling similar to GWs from orbiting binaries. Frequency doubling comes about because after half-cycle of a SASI dipole mode, in which the density distribution roughly undergoes a spatial reversal $\mathbf{x} \rightarrow-\mathbf{x}$, the mass quadrupole moment $\rho\left(x_{i} x_{j}-\delta_{i j} r^{2}\right)$ has already returned to its original value; hence the period of the GW signal is only half the period of the SASI dipole 

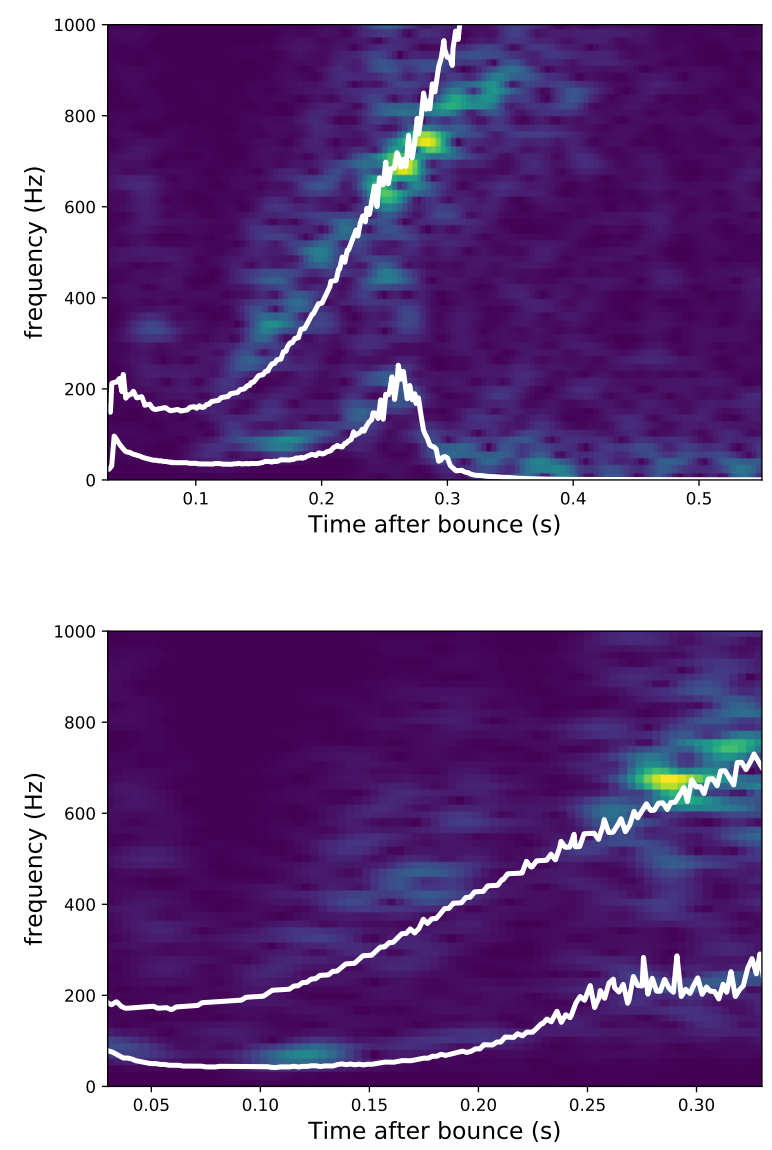

Figure 12. Comparison of the spectrogram of the z85_SFHx model (top), and the z100_SFHx model (bottom)

(same as in Figure 11) and analytic relations for the g-mode and SASI mode frequencies. The lower white curve shows the GW frequencies predicted by Equation 2, accounting for frequency doubling. The white curve at high frequency shows the GW frequency predicted by the Universal relations for the ${ }^{2} g_{2}$-mode from Torres-Forné et al. (2019).

coefficients. Figure 12 illustrates (again for models z85_SFHx and z100_SFHx) that in all our models the SASI emission band is well fit by $2 f_{\text {SASI }}$ up to shock revival.

In the exploding models z85_SFHx and z85_SFHo, the SASI emission band increases in frequency with time up to the point of shock revival and again decreases afterwards as the shock expands. This effect can be seen most clearly in the spectrogram of model z85_SFHx where shock revival occurs later so that the SASI band can reach a frequency of $\sim 200 \mathrm{~Hz}$ before the shock is revived and the GW frequency starts decreasing. As the shock is not revived in the $z 100$ models, their low-frequency emission band continues to increase and reaches a frequency of $\sim 400 \mathrm{~Hz}$ by the end of the simulation. Therefore, in exploding models, the different EoS result in a clear difference in the low-frequency GW emission, but since the connection between the microphysical properties of the EoS and the SASI activity is indirect (through the shock trajectory) and may be compounded by progenitor differences, it is difficult to directly constrain the EoS based on these low-frequency signal features.

Overall, the z85 models with successful shock revival exhibit larger GW amplitudes in line with previous comparisons of GW

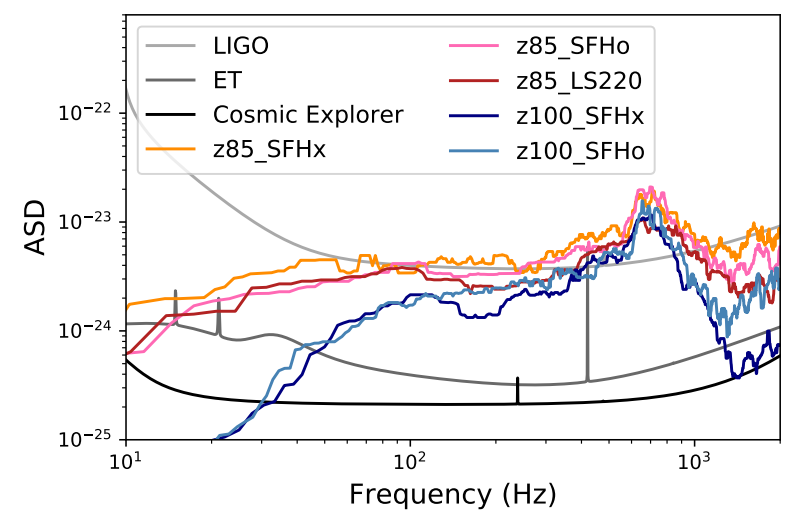

Figure 13. The smoothed amplitude spectral density (ASD) at $50 \mathrm{kpc}$ for the five supernova models compared to the sensitivity curves of current and future GW detectors. The ASD of the models has been computed assuming an observer in the equatorial plane at $(\theta, \varphi)=\left(90^{\circ}, 90^{\circ}\right)$ at a distance of $50 \mathrm{kpc}$. All the models have a maximum amplitude at a frequency of above $\sim 700 \mathrm{~Hz}$.

emission in exploding and non-exploding models. The maximum amplitudes (discarding late-time tail signals) for the $\mathrm{z} 85$ models are $\sim 20 \mathrm{~cm}$, and for the $z 100$ models are $\sim 10 \mathrm{~cm}$. The z85_SFHx and z85_SFHo models develop visible late-time tails, especially model z85_SFHx with a tail amplitude of over $150 \mathrm{~cm}$. The tails are due to anisotropic expansion of the shock wave with a positive amplitude indicating a prolate explosion (Murphy et al. 2009).

\subsection{Detection prospects}

The amplitude spectral density for all our models at a distance of $50 \mathrm{kpc}$ is shown in Figure 13. Our models are in a good frequency range for current ground-based GW detectors and future GW detectors with a similar frequency band such as the Einstein Telescope (Punturo et al. 2010) and Cosmic Explorer (Abbott et al. 2017). The z85 models have stronger low-frequency emission, which will improve their detectability in the Virgo (Acernese \& et al. 2015) and KAGRA (Somiya 2012) GW detectors, which are not as sensitive as LIGO at high frequencies.

We estimate the maximum detectable distance for our models by calculating the matched filter $\operatorname{SNR} \rho$,

$\rho=\frac{\langle s \mid h\rangle^{2}}{\langle h \mid h\rangle}$,

where $h$ is the waveform and $s$ is the data and the inner product is given by

$\langle a \mid b\rangle=4 \int_{0}^{\infty} \frac{a(f) b^{*}(f)}{S_{n}(f)} \mathrm{d} f$,

where $S_{n}$ is the power spectral density (Cutler \& Flanagan 1994). As in previous studies, we assume the threshold SNR for detection at the maximum distance is 8 and that the sensitivity of the detectors at the sources sky position is optimal. Below this threshold value it is assumed that the false alarm rate created by detector noise transients will be too large for a confident detection, although it is not currently possible for us to determine the non-Gaussian features of future detectors noise, and knowledge of the sky position and distance of a CCSNe may increase our ability to detect lower SNR signals. In 
the targeted search for CCSNe during the first and second Observing Runs of LIGO and Virgo, the loudest events had SNRs of $\sim 6$ and false alarm rates that indicate they were consistent with background noise (Abbott et al. 2020). Therefore, we assume an SNR of at least 8 will be needed for a signal to be above the background transient noise.

The results are shown in Table 2 for all models and two different observer directions at $\theta=0^{\circ}$ (pole) and $(\theta, \varphi)=\left(90^{\circ}, 90^{\circ}\right.$ ) (equator). We show the root sum squared GW amplitude $h_{\mathrm{RSS}}$ that would be measured by a GW detector for a source at a distance of $10 \mathrm{kpc}$. It is defined as

$h_{\mathrm{RSS}}=\sqrt{\left(h_{+} F_{+}\right)^{2}+\left(h_{\times} F_{\times}\right)^{2}}$,

where $h_{+}$and $h_{\times}$are the two GW polarisations of the signal. $F_{+}$ and $F_{\times}$are the detectors antenna patterns, which are dependent on the source's sky position. We assume them to be equal to 1 which corresponds to an optimal sky position for the source. All of the models are detectable at Galactic distances in the Advanced LIGO detector. The z85_SFHx and z85_SFHo models have the largest LIGO detection distances with a maximum of $\sim 45 \mathrm{kpc}$. In a network of advanced detectors, it may be possible to detect these model out to the Large Magellanic Cloud at $48 \mathrm{kpc}$. The z100_SFHx model has the smallest detection distance, which is likely an artefact of the short simulation duration, however. As the model has similar amplitudes to model z100_SFHo, we expect the distance for the two models would be similar if the model had been simulated for a longer duration. Since the $\mathrm{z} 100$ models will not collapse to a $\mathrm{BH}$ on short time-scales and may yet explode, the detection distances for these models should be considered lower limits.

The models will be detectable at hundreds of $\mathrm{kpc}$ in the Cosmic Explorer and Einstein Telescope detectors. The z85_SFHx model has detectable distances of up to $515 \mathrm{kpc}$ in Einstein Telescope and $863 \mathrm{kpc}$ in Cosmic Explorer. This indicates that Cosmic Explorer may detect BH-forming stellar collapse in M31 at $778 \mathrm{kpc}$. The detection distances of these models may reach up to a few $\mathrm{Mpc}$ in a multipledetector network of third-generation detectors.

In Table 2, we also show the SNRs in Advanced LIGO for each model at $10 \mathrm{kpc}$, which range from 17 (z100_SFHx) to 37 (z85_SFHx). As we shall see below, such modest SNRs are already enough to spot key features in the time-frequency features of the GW signal. Splitting the contribution to the SNR from frequencies below and above $350 \mathrm{~Hz}$, we find that the high-frequency g-modes are the main component of the total SNR. The direction dependence of the high-frequency and low-frequency contribution to the SNR is modest.

In the light of a more mature understanding of the time-frequency structure of CCSN GW signals, it is increasingly important to not only address the mere question of detectability or the broad-brush distinction of different CCSN explosion scenarios (e.g., Logue et al. 2012; Powell et al. 2016), but also the problem of quantitative parameter estimation. Different from the scenario of rotational collapse (Abdikamalov et al. 2014), quantitative parameter estimation and feature extraction from the post-bounce GW signal is still the subject of active research. Some attempts to extract features from data with realistic noise have already been made by Hayama et al. $(2015,2018)$; Roma et al. (2019), and recently by Bizouard et al. (2020) based on universal relations for PNS oscillations modes. As a complementary approach to model-based parameter estimation it is also insightful to directly consider noisy mock data in the time-frequency domain. In order to construct noisy mock spectrograms, we create Gaussian simulated noise for the Advanced LIGO, Einstein Telescope, and Comic Explorer detectors using the ASD curves shown in Figure 13. To create the detector noise, Gaussian points are drawn in the frequency domain around the ASD curves and are then Fourier transformed to create time domain Gaussian noise. We then add our time domain GW signals to the time domain noise for each detector. Mock spectrograms of signals with LIGO noise at a distance of $5 \mathrm{kpc}$ for an observer in the equatorial plane of the spherical polar grid are shown in the right column of Figure 11.

We find that the characteristic features of the signal, i.e. the g-mode and SASI emission band, remain visible in the spectrograms even at lower SNRs than in Figure 11 down to SNR 20. Even by eye and with second-generation detectors, the SASI frequency could be pinpointed within $\sim 20 \%$ for over $100 \mathrm{~ms}$ and the g-mode frequency within $\sim 10 \%$ at peak emission for a model like z85_SFHx at a distance of $5 \mathrm{kpc}$. With third-generation instruments and a higher SNR by a factor of 10-20, quantitative measurements of mode frequencies will clearly be possible throughout the Milky Way for strong GW emitters. At lower SNR values, where the features are not visible by eye, it may still be possible to extract the features of the signal using waveform reconstruction techniques (Klimenko et al. 2008; Cornish \& Littenberg 2015). Roma et al. (2019) show they can determine that SASI is present in a spectrogram down to SNR values as low as $\sim 10$. Reconstructing the signal modes in time-frequency space will be essential for relating the properties of the detection to the PNS properties and explosion dynamics.

CCSNe are also expected to produce GWs due to the anisotropic neutrino emission (Mueller \& Janka 1997; Kotake et al. 2009; Vartanyan \& Burrows 2020). While this signal component can have amplitudes comparable to the matter signal, it lies at far lower frequencies where the present ground-based GW detectors considered here are not very sensitive.

\section{CONCLUSIONS}

In recent years, a greater understanding of CCSN explosions has been reached through self-consistent $3 \mathrm{D}$ simulations. One of the challenges for 3D CCSN models is now to scan the progenitor parameter space and broadly survey the outcomes of stellar collapse in terms of remnant and explosion properties. More models are also needed to build a more extensive bank of gravitational waveform predictions in order to aid and inform future detections of CCSNe in GWs. In the light of recent GW detections, the final collapse of progenitors in the pulsational pair instability regime and origin of the most massive $\mathrm{BHs}$ produced by $\mathrm{CCSNe}$ are of particular interest and need to be explored more thoroughly by first-principle supernova models.

For this reason, we performed CCSN simulations of two very massive progenitors with the neutrino hydrodynamics code CoCoNuTFMT. The progenitor stars we use are $85 \mathrm{M}_{\odot}$ and $100 \mathrm{M}_{\odot}$ Pop-III stars from the lower end of the pulsational pair instability regime. We used three different nuclear EoS (LS220, SFHx, and SFHo EoS) to examine the EoS sensitivity of the dynamics and GW emission of supernovae from very massive progenitors.

In all of the $85 \mathrm{M}_{\odot}$ models, the shock is revived at relatively early post-bounce times. Because of their very massive cores, these models then form BHs within a few hundreds of milliseconds after shock revival, however. These findings provide further indication for precipitous shock revival in progenitors with high compactness (O'Connor \& Couch 2018; Burrows et al. 2020), which could then develop into fallback supernovae (Chan et al. 2018) with partial envelope ejection or completely collapse to a BH. The model with the LS220 EoS explodes the earliest at $0.17 \mathrm{~s}$ after core-bounce and quickly forms 


\begin{tabular}{cccccccccc}
\hline Progenitor & EoS & $\begin{array}{c}\text { Observer } \\
(\text { position })\end{array}$ & $\begin{array}{c}\text { LIGO } \\
(\mathrm{kpc})\end{array}$ & $\begin{array}{c}\mathrm{ET} \\
(\mathrm{kpc})\end{array}$ & $\begin{array}{c}\mathrm{CE} \\
(\mathrm{kpc})\end{array}$ & $\begin{array}{c}h_{\mathrm{RSS}} @ 10 \mathrm{kpc} \\
\left(10^{-23}\right)\end{array}$ & SNR @ 10 kpc & SNR $_{\text {low } f}$ & SNR \\
\hline z85 $f$ & SFHx & pole & 46 & 479 & 851 & 11.7 & 37 & 18 & 31 \\
\hline z85 & SFHx & equator & 44 & 466 & 825 & 11.6 & 35 & 21 & 28 \\
\hline z85 & SFHo & pole & 44 & 465 & 825 & 11.8 & 36 & 18 & 29 \\
\hline z85 & SFHo & equator & 42 & 427 & 783 & 11.1 & 34 & 18 & 28 \\
\hline z85 & LS220 & pole & 37 & 386 & 690 & 8.51 & 30 & 11 & 27 \\
\hline z85 & LS220 & equator & 30 & 309 & 556 & 7.89 & 24 & 14 & 18 \\
\hline z100 & SFHx & pole & 21 & 216 & 381 & 5.08 & 17 & 8 & 15 \\
\hline z100 & SFHx & equator & 21 & 235 & 408 & 5.34 & 17 & 8 & 16 \\
\hline z100 & SFHo & pole & 24 & 253 & 439 & 6.81 & 19 & 8 & 17 \\
\hline z100 & SFHo & equator & 26 & 282 & 492 & 7.05 & 22 & 11 & 19 \\
\hline
\end{tabular}

Table 2. Summary of GW emission for all models for observers at the pole $\left(\theta=0^{\circ}\right)$ and in the equatorial plane at $(\theta, \varphi)=\left(90^{\circ}, 90^{\circ}\right)$. Columns LIGO, ET and $\mathrm{CE}$ show the maximum distances in kpc based on a threshold matched filtering signal-to-noise ratio (SNR) of 8 in the LIGO, Einstein Telescope, and Cosmic Explorer detectors, respectively. $h_{\mathrm{RSS}}$ is the root-sum-squared amplitude at $10 \mathrm{kpc}$. Column SNR shows the total SNR of the signal in LIGO for a source at $10 \mathrm{kpc}$. The last two columns $\mathrm{SNR}_{\text {low } f}$ and $\mathrm{SNR}_{\text {high } f}$ show the SNR calculated using only the signal at frequencies below and above $350 \mathrm{~Hz}$.

a BH at $0.29 \mathrm{~s}$ after bounce. The model with the SFHx EoS, which supports the highest maximum mass, takes the longest time until shock revival, but is also the last to collapse a $\mathrm{BH}$ and reaches the largest diagnostic energy of $2.7 \times 10^{51} \mathrm{erg}$ due to the longer accretion time. Even in this case, the energy is not sufficient to shed the entire envelope and $\mathrm{BH}$ collapse by fallback is unavoidable. Longer simulations are required to decide whether the incipient explosions lead to partial mass ejection or are eventually stifled. For the most energetic explosion with the SFHx EoS, we estimate a final BH mass in the range of 30.7-34.4 $\mathrm{M}_{\odot}$.

The $100 \mathrm{M}_{\odot}$ models did not explode before the end of the simulation, but heating conditions are already close to runaway shock expansion so that these models would likely explode before $\mathrm{BH}$ collapse. Further simulations will be needed to determine which stars in the pair instability regime will quietly form BHs during their final collapse, and which ones will undergo early or late shock revival before $\mathrm{BH}$ formation and perhaps shed part of the envelope.

We determined the GW emission for all of our models. The GW spectrograms exhibit familiar features with a high-frequency g-mode emission band, and all of the models also have quite strong low-frequency emission. In the SFHx and SFHo models, the lowfrequency emission clearly stems from strong SASI emission. In the $85 \mathrm{M}_{\odot}$ models, the frequency of the SASI emission band increases with time up to the point of shock revival where the SASI disappears. In the $100 \mathrm{M}_{\odot}$ models, the SASI emission band continuously increases in frequency and remains present throughout the simulations even though GW amplitudes decline after $400 \mathrm{~ms}$. The timeintegrated GW spectrum peaks at frequencies of $650-710 \mathrm{~Hz}$, within the sensitivity range of current and third generation GW detectors, which is somewhat higher than the detectors peak sensitivity range, but not unusually high compared to CCSN models of less massive progenitors.

Overall, the GW emission from these very massive progenitors is strong and favourable for detection. We obtain maximum detection distances of up to $46 \mathrm{kpc}$ with Advanced LIGO. Bearing in mind that some of the waveforms are still incomplete, the GW signals from these pulsational pair instability models should be detectable throughout the Galaxy and perhaps in the Large Magellanic Cloud with present-day GW detector networks. The $85 \mathrm{M}_{\odot}$ models with the SFHo and SFHx EoS would be detectable out to M31 in Cosmic Explorer. We demonstrated that the g-mode and SASI emission bands can be identified in noisy spectrograms even by eye for moderately high SNRs of 20, which are easily reached for events in the Milky Way and its satellites in third-generation instruments. This underscores the potential for measuring the dynamics of quiet $\mathrm{BH}$ collapse or weak explosions with GWs in the next decades.

To date there are some potential candidates for pulsational pair instability supernovae but no confirmed observations (Arcavi et al. 2017; Woosley 2018; Gomez et al. 2019). Some theoretical studies have made predictions of a lower limit on the rate of pulsational pair instability supernovae of $\sim 0.1 \mathrm{Gpc}^{-3} \mathrm{yr}^{-1}$ at redshift zero (Stevenson et al. 2019). Pulsational pair instability supernovae are unlikely to occur in our Galaxy, as they are only expected to occur in low metallicity environments, however it is possible they may be detected in the local group by the next generation of GW detectors.

\section{ACKNOWLEDGEMENTS}

We thank KaHo Tse for providing the code for the periodogram used to analyse the pre-supernova evolution of model z85. We thank Kei Kotake for helpful comments. The authors are supported by the Australian Research Council (ARC) Centre of Excellence (CoE) for Gravitational Wave Discovery (OzGrav) project number CE170100004. JP is supported by the ARC Discovery Early Career Researcher Award (DECRA) project number DE210101050. BM is supported by ARC Future Fellowship FT160100035. AH is supported by the ARC CoE for All Sky Astrophysics in 3 Dimensions (ASTRO 3D) project number CE170100013. We acknowledge computer time allocations from Astronomy Australia Limited's ASTAC scheme and the National Computational Merit Allocation Scheme (NCMAS). Some of this work was performed on the Gadi supercomputer with the assistance of resources and services from the National Computational Infrastructure (NCI), which is supported by the Australian 
Government. Some of this work was performed on the OzSTAR national facility at Swinburne University of Technology. OzSTAR is funded by Swinburne University of Technology and the National Collaborative Research Infrastructure Strategy (NCRIS).

\section{DATA AVAILABILITY}

The data from our simulations will be made available upon reasonable requests made to the authors.

\section{REFERENCES}

Abbott B. P., et al., 2017, Classical and Quantum Gravity, 34, 044001

Abbott B. P., et al., 2018, Phys. Rev. Lett., 121, 161101

Abbott B. P., et al., 2020, Phys. Rev. D, 101, 084002

Abdikamalov E., Gossan S., DeMaio A. M., Ott C. D., 2014, Phys. Rev. D, 90,044001

Acernese F., et al. 2015, Classical and Quantum Gravity, 32, 024001

Ackley K., et al., 2020, arXiv e-prints, p. arXiv:2007.03128

Adams S. M., Kochanek C. S., Gerke J. R., Stanek K. Z., Dai X., 2017a, MNRAS, 468, 4968

Adams S. M., Kochanek C. S., Gerke J. R., Stanek K. Z., 2017b, MNRAS, 469,1445

Andresen H., Müller B., Müller E., Janka H. T., 2017, MNRAS, 468, 2032

Andresen H., Müller E., Janka H. T., Summa A., Gill K., Zanolin M., 2019, MNRAS, 486, 2238

Andresen H., Glas R., Janka H.-T., 2020, arXiv e-prints, p. arXiv:2011.10499

Arcavi I., et al., 2017, Nature, 551, 210

Astone P., Cerdá-Durán P., Di Palma I., Drago M., Muciaccia F., Palomba C., Ricci F., 2018, Phys. Rev. D, 98, 122002

Barkat Z., Rakavy G., Sack N., 1967, Phys. Rev. Lett., 18, 379

Belczynski K., et al., 2016, A\&A, 594, A97

Bizouard M.-A., Maturana-Russel P., Torres-Forné A., Obergaulinger M., Cerdá-Durán P., Christensen N., Font J. A., Meyer R., 2020, arXiv eprints, p. arXiv:2012.00846

Blanchet L., Damour T., Schaefer G., 1990, MNRAS, 242, 289

Blondin J. M., Mezzacappa A., 2006, ApJ, 642, 401

Blondin J. M., Mezzacappa A., DeMarino C., 2003, The Astrophysical Journal, 584, 971

Bollig R., Janka H.-T., Lohs A., Martínez-Pinedo G., Horowitz C. J., Melson T., 2017, Physical Review Letters, 119, 242702

Bollig R., Yadav N., Kresse D., Janka H. T., Mueller B., Heger A., 2020, arXiv e-prints, p. arXiv:2010.10506

Bruenn S. W., et al., 2020, ApJS, 248, 11

Buras R., Rampp M., Janka H.-T., Kifonidis K., 2006, A\&A, 447, 1049

Burrows A., Vartanyan D., 2020, arXiv e-prints, p. arXiv:2009.14157

Burrows A., Dolence J. C., Murphy J. W., 2012, ApJ, 759, 5

Burrows A., Radice D., Vartanyan D., Nagakura H., Skinner M. A., Dolence J. C., 2020, MNRAS, 491, 2715

Capano C. D., et al., 2020, Nature Astronomy, 4, 625

Chan C., Müller B., Heger A., Pakmor R., Springel V., 2018, ApJ, 852, L19

Chan C., Müller B., Heger A., 2020, MNRAS, 495, 3751

Chen K.-J., Heger A., Woosley S., Almgren A., Whalen D. J., 2014, ApJ, 792,44

Cornish N. J., Littenberg T. B., 2015, Classical and Quantum Gravity, 32, 135012

Cutler C., Flanagan É. E., 1994, Phys. Rev. D, 49, 2658

Dimmelmeier H., Font J. A., Müller E., 2002, A\&A, 393, 523

Dimmelmeier H., Ott C. D., Marek A., Janka H. T., 2008, Phys. Rev. D, 78, 064056

Ertl T., Janka H.-T., Woosley S. E., Sukhbold T., Ugliano M., 2016, ApJ, 818, 124

Finn L. S., 1989, in Evans C. R., Finn L. S., Hobill D. W., eds, Frontiers in Numerical Relativity. Cambridge University Press, Cambridge (UK), pp $126-145$
Finn L. S., Evans C. R., 1990, ApJ, 351, 588

Foglizzo T., Galletti P., Scheck L., Janka H.-T., 2007, ApJ, 654, 1006

Fowler W. A., Hoyle F., 1964, ApJS, 9, 201

Fryer C. L., Woosley S. E., Heger A., 2001, ApJ, 550, 372

Fuller J., Klion H., Abdikamalov E., Ott C. D., 2015, MNRAS, 450, 414

Furusawa S., Togashi H., Nagakura H., Sumiyoshi K., Yamada S., Suzuki H., Takano M., 2017, Journal of Physics G Nuclear Physics, 44, 094001

Gerke J. R., Kochanek C. S., Stanek K. Z., 2015, MNRAS, 450, 3289

Gomez S., et al., 2019, ApJ, 881, 87

Hayama K., Kuroda T., Kotake K., Takiwaki T., 2015, Phys. Rev. D, 92, 122001

Hayama K., Kuroda T., Kotake K., Takiwaki T., 2018, MNRAS, 477, L96

Heger A., Woosley S. E., 2002, ApJ, 567, 532

Heger A., Woosley S. E., 2010, ApJ, 724, 341

Heger A., Fryer C. L., Woosley S. E., Langer N., Hartmann D. H., 2003, ApJ, 591,288

Heger A., Cumming A., Woosley S. E., 2007, ApJ, 665, 1311

Hüdepohl L., 2014, PhD thesis, Technical University of Munich, https: //mediatum.ub.tum.de/1177481

Janka H.-T., 2001, A\&A, 368, 527

Janka H.-T., 2012, Annual Review of Nuclear and Particle Science, 62, 407

Klimenko S., Yakushin I., Mercer A., Mitselmakher G., 2008, Classical and Quantum Gravity, 25, 114029

Kotake K., Iwakami W., Ohnishi N., Yamada S., 2009, ApJ, 697, L133

Kozyreva A., et al., 2017, MNRAS, 464, 2854

Kuroda T., Kotake K., Takiwaki T., 2016, ApJ, 829, L14

Kuroda T., Kotake K., Hayama K., Takiwaki T., 2017, ApJ, 851, 62

Kuroda T., Kotake K., Takiwaki T., Thielemann F.-K., 2018, Monthly Notices of the Royal Astronomical Society: Letters, 477, L80-L84

Landfield R. E., 2018, PhD thesis, University of Tennesse, Knoxville, https://trace. tennessee.edu/cgi/viewcontent.cgi? article $=6838 \&$ context $=$ utk_graddiss

Landry P., Essick R., Chatziioannou K., 2020, Phys. Rev. D, 101, 123007

Lattimer J. M., Swesty F. D., 1991, Nucl. Phys., A535, 331

Linial I., Fuller J., Sari R., 2020, arXiv e-prints, p. arXiv:2011.12965

Liu J., et al., 2019, Nature, 575, 618

Logue J., Ott C. D., Heng I. S., Kalmus P., Scargill J. H. C., 2012, Phys. Rev. D, 86, 044023

Mandel I., Müller B., 2020, MNRAS, 499, 3214

Matzner C. D., Ro S., 2020, arXiv e-prints, p. arXiv:2011.08861

Mezzacappa A., et al., 2020, Phys. Rev. D, 102, 023027

Moriya T. J., Müller B., Chan C., Heger A., Blinnikov S. I., 2019, ApJ, 880, 21

Morozova V., Radice D., Burrows A., Vartanyan D., 2018, ApJ, 861, 10

Mueller E., Janka H. T., 1997, A\&A, 317, 140

Müller B., 2019, Annual Review of Nuclear and Particle Science, 69, 253

Müller B., 2020, Living Reviews in Computational Astrophysics, 6, 3

Müller B., Janka H.-T., 2014, ApJ, 788, 82

Müller B., Janka H.-T., 2015, MNRAS, 448, 2141

Müller B., Janka H.-T., Dimmelmeier H., 2010, ApJS, 189, 104

Müller E., Janka H. T., Wongwathanarat A., 2012, A\&A, 537, A63

Müller B., Janka H.-T., Marek A., 2013, ApJ, 766, 43

Müller B., et al., 2019, MNRAS, 484, 3307

Murphy J. W., Ott C. D., Burrows A., 2009, ApJ, 707, 1173

O'Connor E. P., Couch S. M., 2018, ApJ, 865, 81

O'Connor E., Ott C. D., 2011, ApJ, 730, 70

Ott C. D., Roberts L. F., da Silva Schneider A., Fedrow J. M., Haas R., Schnetter E., 2018, ApJ, 855, L3

Paczynski B., 1983, ApJ, 264, 282

Pan K.-C., Liebendörfer M., Couch S. M., Thielemann F.-K., 2018, ApJ, 857, 13

Pan K.-C., Liebendörfer M., Couch S., Thielemann F.-K., 2020, arXiv eprints, p. arXiv:2010.02453

Powell J., Müller B., 2019, MNRAS, 487, 1178

Powell J., Müller B., 2020, MNRAS, 494, 4665

Powell J., Gossan S. E., Logue J., Heng I. S., 2016, Phys. Rev. D, 94, 123012

Punturo M., et al., 2010, Classical and Quantum Gravity, 27, 194002

Raaijmakers G., et al., 2020, The Astrophysical Journal, 893, L21 
Radice D., Morozova V., Burrows A., Vartanyan D., Nagakura H., 2019, ApJ, 876, L9

Rampp M., Janka H.-T., 2002, A\&A, 396, 361

Rauscher T., Heger A., Hoffman R. D., Woosley S. E., 2002, ApJ, 576, 323

Richers S., Ott C. D., Abdikamalov E., O'Connor E., Sullivan C., 2017, Phys. Rev. D, 95, 063019

Roma V., Powell J., Heng I. S., Frey R., 2019, Phys. Rev. D, 99, 063018

Shen H., Toki H., Oyamatsu K., Sumiyoshi K., 1998, Nuclear Phys. A, 637, 435

Shibagaki S., Kuroda T., Kotake K., Takiwaki T., 2020, MNRAS, 493, L138

Shibagaki S., Kuroda T., Kotake K., Takiwaki T., 2021, MNRAS,

Smartt S. J., 2015, Publ. Astron. Soc. Australia, 32, e016

Somiya K., 2012, Classical and Quantum Gravity, 29, 124007

Sotani H., Takiwaki T., 2020, MNRAS, 498, 3503

Sotani H., Kuroda T., Takiwaki T., Kotake K., 2017, Phys. Rev. D, 96, 063005

Steiner A. W., Hempel M., Fischer T., 2013, ApJ, 774, 17

Stevenson S., Sampson M., Powell J., Vigna-Gómez A., Neijssel C. J., Szécsi D., Mandel I., 2019, ApJ, 882, 121

Takiwaki T., Kotake K., 2018, MNRAS, 475, L91

Tews I., Lattimer J. M., Ohnishi A., Kolomeitsev E. E., 2017, ApJ, 848, 105

The LIGO Scientific Collaboration Aasi J., Abbott B. P., Abbott R., et al. 2015, Classical and Quantum Gravity, 32, 074001

The LIGO Scientific Collaboration the Virgo Collaboration Abbott R., Abbott T. D., Abraham S., Acernese F., Ackley K., et al., 2020, arXiv e-prints, p. arXiv:2009.01075

Torres-Forné A., Cerdá-Durán P., Passamonti A., Font J. A., 2018, MNRAS, 474,5272

Torres-Forné A., Cerdá-Durán P., Obergaulinger M., Müller B., Font J. A., 2019, Phys. Rev. Lett., 123, 051102

Tse K., Galloway D. K., Chou Y., Heger A., Hsieh H.-E., 2021, MNRAS, 500,34

Vartanyan D., Burrows A., 2020, ApJ, 901, 108

Walk L., Tamborra I., Janka H.-T., Summa A., Kresse D., 2020, Phys. Rev. D, 101,123013

Weaver T. A., Zimmerman G. B., Woosley S. E., 1978, ApJ, 225, 1021

Woosley S. E., 2017, ApJ, 836, 244

Woosley S. E., 2018, ApJ, 863, 105

Woosley S. E., Heger A., Weaver T. A., 2002, Reviews of Modern Physics, 74, 1015

Woosley S. E., Blinnikov S., Heger A., 2007, Nature, 450, 390

Yasin H., Schäfer S., Arcones A., Schwenk A., 2020, Phys. Rev. Lett., 124, 092701

da Silva Schneider A., O'Connor E., Granqvist E., Betranhandy A., Couch S. M., 2020, ApJ, 894, 4

This paper has been typeset from a $\mathrm{T}_{\mathrm{E}} \mathrm{X} / \mathrm{L} \mathrm{T} \mathrm{E} \mathrm{X}$ file prepared by the author. 